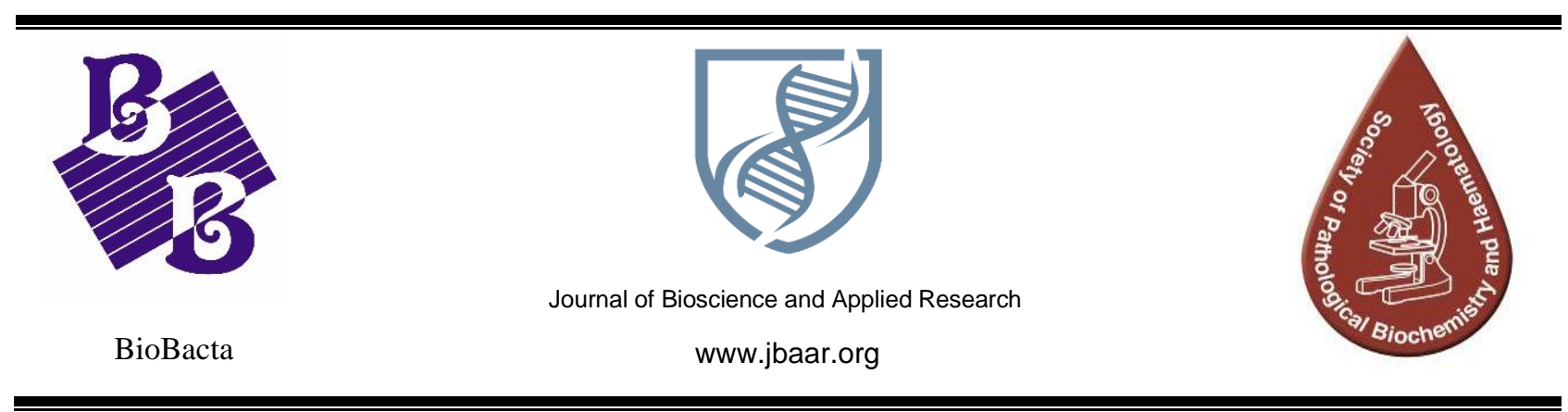

\title{
Synthesis of Thiophenyl, Thiazolyl, and Pyridinyl Androstane Derivatives: A Novel Class of Potent Anti-inflammatory and Anti-ulcerogenic Heterocyclic Steroids
}

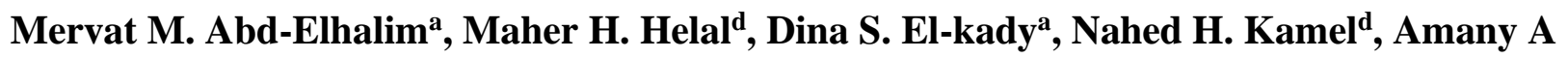 \\ Sleem $^{b}$, Omar M.E. Abdel-Salam ${ }^{c}$, Amira E. M. Abdallah ${ }^{d}$, Gamal A. Elmegeed ${ }^{\mathrm{a} *}$
}

${ }^{\text {a}}$ Hormones Dept., ${ }^{\text {bPharmacology Dept., }}{ }^{\text {cT}}$ Toxicology and Narcotics Dept., Medical Research Division, National Research Centre, Dokki, Giza-12622, Egypt, ${ }^{\mathrm{d}}$ Chemistry Department, Faculty of Science, Helwan University, Ain Helwan, Cairo-

11795, Egypt

*Corresponding author details: Prof: Gamal A. Elmegeed

Hormones Department, National Research Centre, 12622 Dokki, Cairo, Egypt

Tel: +2 02 33872607, Fax: +2 02 33370931,E-mail: gamalae@ hotmail.com, https://orcid.org/0000-0003-1914-1543

Received: October 25, 2019; Accepted: November 25, 2019

DOI: $10.21608 /$ jbaar.2019.115401

\begin{abstract}
:
The investigation of drugs used for the treatment of inflammatory diseases with limited side effects becomes an urgent need for inflammation patients. This search aimed at the preparation of novel hybrid heterosteroids with structures especially non-ulcerogenic and anti-inflammatory activities. The heterocyclic steroids were formulated using simple and effective techniques. IR, ${ }^{1} \mathrm{H}$ NMR, ${ }^{13} \mathrm{C}$ NMR spectra and elemental microanalysis were used to characterize the synthesized compound. The in vivo anti-inflammatory activity of some of these compounds was studied using carrageenan-induced paw oedema assay. Also, the effect of the different compounds on the development of gastric mucosal damage induced in rats by $96 \%$ EtOH administration was studied. The most marked and sustained inhibition of the oedema response was observed with the administration of the low and high doses (25 and $50 \mathrm{mg} / \mathrm{kg}$ ) of compounds $\mathbf{8 , 4}, \mathbf{2 3}, \mathbf{1 4}$ as well as by the high dose of compound 32. There was no significant difference in the degree of oedema inhibition between the low and high doses of compounds $\mathbf{8 , 2 3}, \mathbf{1 4}$ at all-time points in the study. All investigated compounds administered at $(50 \mathrm{mg} / \mathrm{kg})$ dose inhibited gastric mucosal lesions induced in rats by 96\% EtOH administration. Compounds 4, 9, 11, 26, 32 were the most effective in inhibiting lesion formation. No lesions were observed after the administration of compounds $\mathbf{9}, 26$ at $50 \mathrm{mg} / \mathrm{kg}$. These findings are approaching a distinctive chance to create fresh anti-inflammatory drugs that eliminate the ulcerogenic liabilities connected with drugs presently on the market.
\end{abstract}

Keywords: Anti-inflammatory, Anti-ulcerogenic, Heterocycles-Steroids, Synthesis 


\section{Introduction}

The inflammatory illness affects millions of individuals worldwide, leading to financial loss and mortality, as well as inflammatory lung illnesses such as asthma, chronic obstructive pulmonary disease and other illnesses, including allergic rhinitis, rheumatoid arthritis, osteoarthritis, inflammatory intestinal illnesses, and psoriasis. Drug companies and biotechnology businesses spend billions of dollars to define and create advanced therapies for the treatment of such illnesses [ Soni et al., 2011]. Inflammatory diseases are widely prevalent throughout the world [Kozuch et al., 2008]. Drugs used to treat severe and chronic inflammatory diseases inhibit biological mechanisms that relate to inflammation occurrence and disorders. Our study has concentrated in the latest years on designing, synthesizing and pharmacologically evaluating novel molecules with anti-inflammatory, antinociceptive and analgesic, activities as prospective secure and efficient medications [Elmegeed et al., 2005; Elmegeed et al., 2007; Elmegeed et al., 2010].

Due to their broad range of unique biological activities, steroids have drawn a lot of attention. They can control a range of biological procedures and can, therefore, be used as medicinal products to treat several diseases [Lipworth 1999; Naumann et al., 2011]. A lot of attention was paid to newly established steroids. Their preparation is a stimulating task for the organic chemist, which often requires fresh and usually helpful responses to develop. It has also been shown that the biological characteristics of altered steroids are of concern. In recent years, the interest of steroid research has been concentrated in part upon the synthesis of unusual steroid structures with the aim of finding new physiologically active compounds [Albuquerque et al., 2019; Ledneczki et al., 2019]. Developing novel heterocyclic derivatives and spreading the range of their biological activity is the main target of steroid chemistry. Modification of steroids by attaching of heterocyclic rings often leads to a change of their physiological efficacy and the occurrence of new interesting biological and pharmacological properties [Tantawy et al., 2019; Ansari et al., 2019; Frank et al., 2013].

One of the most important issues was the synthesis and biological study of many heterocyclic steroids as extremely potent anti-inflammatory agents [Elmegeed et al., 2005, Mohareb et al., 2011; Cai et al., 2019]. The development of an efficient curative agent for inflammation treatment has experienced continuous evolution leading to the creation of more efficient types of drugs [Elgazwy et al., 2012]. Hybridization of pharmacophores on the same scaffold is a well-established approach to drug synthesis [Pillai et al., 2003; Venkatachalam et al., 2006]. Based on the above mentioned our aim was to synthesize new hybrid derivatives by modification of steroid moiety with heterocyclic rings and tested the anti-inflammatory and antiulcerogenic activities of the novel steroidal heterocyclic derivatives with the most structurally promising rat model.

\section{Materials and Methods}

\subsection{Synthetic methods, analytical and spectral data}

Starting epi-androsterone was purchased from Sigma Company, USA. All solvents were hydrated by distillation prior to use. All melting points were measured using an electrothermal apparatus and are uncorrected. The IR spectra were recorded in ( $\mathrm{KBr}$ discs) on Shimadzu FT-IR 8201 PC spectrometer and expressed in $\mathrm{cm}^{-1}$. The ${ }^{1} \mathrm{H}$ NMR and ${ }^{13} \mathrm{C}$ NMR spectra were recorded with Jeol instrument (Japan), at 270 and $125 \mathrm{MHz}$ respectively, in DMSO- $\mathrm{d}_{6}$ as a solvent and chemical shifts were recorded in ppm relative to TMS. The spin multiplicities were abbreviated by the letters: ssinglet, d-doublet, t-triplet, quartet and m (multiplet, more than quartet). Mass spectra were recorded on a GCMS-QP 1000 ex spectra mass spectrometer operating at $70 \mathrm{EV}$. Elemental analyses were carried by the Micro-analytical Data Unit at the National Research Centre, Giza, Egypt and the microanalytical Data Unit at Cairo University, Giza, 
Egypt. The reactions were monitored by thin-layer chromatography (TLC) which was carried out using Merck 60 F254 aluminum sheets and visualized by UV light $(254 \mathrm{~nm})$. The mixtures were separated by preparative TLC and gravity chromatography. All steroid derivatives showed the characteristic spectral data of cyclopentanoperhydrophenanthrene nuclei of androstane series were similar to those reported in literature [Fuente et al., 2005].

\subsubsection{Preparation of compounds $4 \& 5$}

To a mixture of cyanoacetylhydrozonoandrostane (1) $(0.385 \mathrm{~g}, 1 \mathrm{~m} \mathrm{~mol})$ and sulphur $(0.032 \mathrm{~g}, 1 \mathrm{~m} \mathrm{~mol})$ in dioxane (30) $\mathrm{mL}$, equimolar amount of ethyl cyanoacetate $2(0.113 \mathrm{~g}, 1 \mathrm{~m} \mathrm{~mol})$ or malononitrile 3 $(0.069 \mathrm{~g}, 1 \mathrm{~m} \mathrm{~mol})$ was added followed by few drops of trimethylamine. The reaction mixture, in each case, was heated under reflux for 3-5 hr. leave the reaction mixture until cooling, poured on ice/water mixture, neutralized with dilute hydrochloric acid. The solid product obtained upon cooling was collected by filtration and crystallized from absolute ethanol.

Ethyl (Z)-5-(2-(3-acetoxy-10, 13-

dimethylhexadecahydro-17H-

cyclopenta[a]phenanthren-17-ylidene) hydrazine-

1-carbonyl)-2,4-diaminothiophene-3-carboxylate

(4).

Yellow crystals from absolute ethanol, yield $0.42 \mathrm{~g}$ (75\%); mp 238-240 ${ }^{\circ} \mathrm{C}$; IR $\left(\mathrm{KBr}, \mathrm{cm}^{-1}\right)$ : v $3445-$ $3350\left(\mathrm{NH}_{2}, \mathrm{NH}\right), 2939,2853\left(\mathrm{CH}_{3}, \mathrm{CH}_{2}\right), 1732$ $(\mathrm{C}=\mathrm{O}), \quad 1656 \quad(\mathrm{C}=\mathrm{N}), \quad 1468 \quad(\mathrm{C}=\mathrm{C}) . \quad{ }^{1} \mathrm{H} \quad \mathrm{NMR}$ (DMSO- $\left.d_{6}, \mathrm{ppm}\right): \delta=0.81\left(\mathrm{~s}, 3 \mathrm{H}, 19-\mathrm{CH}_{3}\right), 1.06(\mathrm{~s}$, $3 \mathrm{H}, 18-\mathrm{CH}_{3}$ ), , 1.32 (t, 3H, $\mathrm{CH}_{3}$-ester), $3.06(\mathrm{~m}, 1 \mathrm{H}$, C5- $\alpha \mathrm{H}$ ), 4.57(q, 2H, $\mathrm{CH}_{2}$-ester), 5.80 (s, 2H, $\mathrm{NH}_{2}$, $\mathrm{D}_{2} \mathrm{O}$ - exchangeable), $7.40\left(\mathrm{~s}, 2 \mathrm{H}, \mathrm{NH}_{2}, \mathrm{D}_{2} \mathrm{O}-\right.$ exchangeable ), $10.80 \quad\left(\mathrm{~s}, \quad 1 \mathrm{H}, \quad \mathrm{NH}, \quad \mathrm{D}_{2} \mathrm{O}-\right.$ exchangeable). ${ }^{13} \mathrm{C}$ NMR (DMSO- $\left.d_{6}, \mathrm{ppm}\right) \delta=39.8$ (C-1), 24.4 (C-2), 40,6 (C-3), 30.8 (C-4), 39.6 (C-5), 27.6 (C-6), 31.8 (C-7), 34.9 (C-8), 51.1 (C-9), 35.7 (C-10), 21.8 (C-11), 34.1 (C-12), 44.5 (C-13), 73.3 (C-14), 34.1 (C- 15), 26.9 (C-16), 166.2 (C-17), 23.9 (C-18), 20.6 (C-19), 170.3 (CO, acetate), $53.2\left(\mathrm{CH}_{3}\right.$ acetate), $160.0(\mathrm{C}=\mathrm{O}), \mathrm{MS}(\mathrm{EI}) \mathrm{m} / \mathrm{z}(\%): 558\left(\mathrm{M}^{+}\right.$,
31\%), 451 (30), 467 (20), 317 (60), 218 (10), 159 (18). Calcd. for $\mathrm{C}_{29} \mathrm{H}_{42} \mathrm{~N}_{4} \mathrm{O}_{5} \mathrm{~S}$ (558.74): C, 62.34; H, 7.58; N, 10.03; S, $5.74 \%$, Found: C, 62.49; H, 7.71; N, 10.26; S, 5.98\%.

\section{(Z)-17-(2-(3,5-diamino-4-cyanothiophene-2-}

carbonyl)hydrazineylidene)-10,13-

\section{dimethylhexadecahydro-1H-}

cyclopenta[a]phenanthren-3-yl acetate (5).

Brown crystals from absolute ethanol, yield $0.35 \mathrm{~g}$ (68\%) , mp 186-188 ${ }^{\circ} \mathrm{C}$; IR $\left(\mathrm{KBr}, \mathrm{cm}^{-1}\right)$ : v 3463$3422\left(\mathrm{NH}_{2}-\mathrm{NH}\right), 2939,2853\left(\mathrm{CH}_{3}, \mathrm{CH}_{2}\right), 2208$ $(\mathrm{CN}), 1730(\mathrm{C}=\mathrm{O}), 1645(\mathrm{C}=\mathrm{N}), 1468(\mathrm{C}=\mathrm{C}) .{ }^{1} \mathrm{H}$ NMR (DMSO- $\left.d_{6}, \mathrm{ppm}\right): \delta=0.80\left(\mathrm{~s}, 3 \mathrm{H}, 19-\mathrm{CH}_{3}\right)$, 1.16 (s, 3H, 18- $\left.\mathrm{CH}_{3}\right), 2.50$ (s, 3H, $\left.\mathrm{CH}_{3} \mathrm{CO}\right), 3.06$ (m, $1 \mathrm{H}, \mathrm{C} 5-\alpha \mathrm{H}), 6.85$ (s, $2 \mathrm{H}, \mathrm{NH}_{2}, \mathrm{D}_{2} \mathrm{O}$-exchangeable), 7.59 (s, $2 \mathrm{H}, \mathrm{NH}_{2}, \mathrm{D}_{2} \mathrm{O}$-exchangeable ), $9.78(\mathrm{~s}, 1 \mathrm{H}$, $\mathrm{NH}, \mathrm{D}_{2} \mathrm{O}$-exchangeable). ${ }^{13} \mathrm{C}$ NMR (DMSO- $d_{6}$, ppm): $\delta=37.7$ (C-1), 27.3, (C-2), 37.5 (C-3), 34.5 (C-4), 43.0 (C-5), 27.1 (C-6), 31.0 (C-7), 34.3 (C-8), 53.0 (C-9), 35.0 (C-10), 4.1 (C-11), 31.1 (C-12), 36.3 (C-13), 63.3 (C-14), 33.9 (C- 15), 31.0 (C-16), 166.3 (C-17), 23.7 (C-18), 12.2 (C-19), 170.2 (CO, acetate $), 115.3(\mathrm{CN}), 169.0(\mathrm{C}=\mathrm{O}), 21.00 \quad\left(\mathrm{CH}_{3}\right.$ acetate), $132.4,157.00(\mathrm{C}=\mathrm{C}$ aromatic carbons). $\mathrm{MS}$ (EI) m/z (\%): $511\left(\mathrm{M}^{+}, 29 \%\right), 383$ (36), 246 (32), 201 (32). Calcd. for $\mathrm{C}_{27} \mathrm{H}_{37} \mathrm{~N}_{5} \mathrm{O}_{3} \mathrm{~S}$ (511.69) C, 63.38; H, 7.29; N, 13.69; S, 6.27\%, Found: C, 63.56; H, $7.45 ; \mathrm{N}, 13.57 ; \mathrm{S}, 6.02 \%$.

\subsubsection{Preparation of compounds of compounds $8 \& 9$}

To a mixture of compound $\mathbf{1}(0.385 \mathrm{~g}, 1 \mathrm{~m} \mathrm{~mol})$, malononitrile $6(0.069 \mathrm{~g}, 1 \mathrm{~m} \mathrm{~mol})$, or ethyl cyanoacetate $7(0.113 \mathrm{~g}, 1 \mathrm{~m} \mathrm{~mol})$, in dioxane (30 $\mathrm{mL}$ ), three drops TEA was added. The reaction mixture was heated under reflux for $3 \mathrm{hr}$ until all the reactants had disappeared as indicated by TLC. Leave the mixture until cooling, poured on crushed ice/water mixture, neutralized with hydrochloric acid. The solid product that formed was collected by filtration, crystallized from absolute ethanol.

(E)-17-((4,6-diamino-5-cyano-2-oxopyridin-1(2H)yl)imino)-10,13-dimethylhexadecahydro-1Hcyclopenta[a]phenanthren-3-yl acetate (8). 
Pale yellow crystals from absolute ethanol, yield $0.21 \mathrm{~g}(44 \%)$; mp 130-135 ${ }^{\circ} \mathrm{C}$; IR $\left(\mathrm{KBr}, \mathrm{cm}^{-1}\right): \mathrm{V}$ $3437\left(\mathrm{NH}_{2}\right), 2935,2855\left(\mathrm{CH}_{3}, \mathrm{CH}_{2}\right), 2205(\mathrm{CN})$, $1737(\mathrm{C}=\mathrm{O}), 1656(\mathrm{C}=\mathrm{N}), 1468(\mathrm{C}=\mathrm{C}) .{ }^{1} \mathrm{H}$ NMR (DMSO- $\left.d_{6}, \mathrm{ppm}\right): \delta=0.77\left(\mathrm{~s}, 3 \mathrm{H}, 19-\mathrm{CH}_{3}\right), 1.09$ (s, $\left.3 \mathrm{H}, 18-\mathrm{CH}_{3}\right), 2.02\left(\mathrm{~s}, 3 \mathrm{H}, \mathrm{CH}_{3} \mathrm{CO}\right), 3.96(\mathrm{~m}, 1 \mathrm{H}$, C5- $\alpha \mathrm{H}$ ), 6.42 (s, 2H, NH $\mathrm{N}_{2} \mathrm{O}$-exchangeable), 8.21 (s, 2H, $\mathrm{NH}_{2}, \mathrm{D}_{2} \mathrm{O}$-exchangeable). ${ }^{13} \mathrm{C}$ NMR (DMSO$\left.d_{6}, \mathrm{ppm}\right): \delta=36.1(\mathrm{C}-1), 27.1(\mathrm{C}-2), 73.6$ (C-3), 44.3 (C-4), 39.5 (C-5), 27.0 (C-6), 31.1 (C-7), 32.7 (C8), 53.7 (C-9), 36.7 (C-10), 20.9 (C-11), 33.1 (C12), 42.8 (C-13), 63.3 (C-14), 23.9 (C- 15), 26.21 (C-16), 166.2 (C-17), 23.7 (C-18), 12.2 (C19),145.3,83.1(C=C), $160.0 \quad(\mathrm{C}=\mathrm{O}), 115.8 \quad(\mathrm{CN})$, 69.9,177.9 $(\mathrm{C}=\mathrm{C}), 175.9(\mathrm{C}=\mathrm{O}), 52.2\left(\mathrm{CH}_{3}\right) . \mathrm{MS}$ (EI): m/z (\%): $479\left(\mathrm{M}^{+}, 29 \%\right), 453$ (3), 438 (40), 330 (7), 163 (22), 149 (20). Calcd. for $\mathrm{C}_{27} \mathrm{H}_{37} \mathrm{~N}_{5} \mathrm{O}_{3}$ (479.63): C, 67.61; H, 7.78; N, 14.60\%, Found: C, $67.89 ; \mathrm{H}, 7.99 ; \mathrm{N}, 14.78 \%$.

\section{Ethyl (E)-1-((3-acetoxy-10,13-}

dimethylhexadecahydro-17H-

cyclopenta[a]phenanthren-17-ylidene)amino)-2,4diamino-6-oxo-1,2-dihydropyridine-3-carboxylate (9).

Dark brown crystals from absolute ethanol, yield $0.24 \mathrm{~g}(45 \%) ; \mathrm{mp} 185-187{ }^{\circ} \mathrm{C}$; IR $\left(\mathrm{KBr}, \mathrm{cm}^{-1}\right)$ : v $3462\left(\mathrm{NH}_{2}\right), 2928,2856\left(\mathrm{CH}_{3}, \mathrm{CH}_{2}\right), 1729(\mathrm{C}=\mathrm{O})$, $1636(\mathrm{C}=\mathrm{N}), 1468(\mathrm{C}=\mathrm{C}) .{ }^{1} \mathrm{H}$ NMR (DMSO- $d_{6}$, ppm): $\delta=0.76\left(\mathrm{~s}, 3 \mathrm{H}, 19-\mathrm{CH}_{3}\right), 1.19(\mathrm{~s}, 3 \mathrm{H}, 18-$ $\mathrm{CH}_{3}$ ), 1.21 (t, 3H, $\mathrm{CH}_{3}$ ester), 2.49 (s, 3H, $\mathrm{CH}_{3} \mathrm{CO}$ ), 4.21 (q, 2H, $\mathrm{CH}_{2}$-ester), 4.79 (s ,2H, $\mathrm{NH}_{2}, \mathrm{D}_{2} \mathrm{O}-$ exchangeable $), \quad 6.02 \quad\left(\mathrm{~s}, \quad 2 \mathrm{H}, \quad \mathrm{NH}_{2} \quad \mathrm{D}_{2} \mathrm{O}-\right.$ exchangeable). ${ }^{13} \mathrm{C}$ NMR (DMSO- $\left.d_{6}, \mathrm{ppm}\right): \delta=38.9$ (C-1), 25.1 (C-2), 40.9 (C-3), 36.9 (C-4), 42.6 (C-5), 27.2 (C-6), 30.9 (C-7), 34.8 (C-8), 53.1 (C-9), 35.7 (C-10), 21.2 (C-11), 31.1 (C-12), 42.1 (C-13), 63.2 (C-14), 33.8 (C-15), $26.2(\mathrm{C}-16), 166.1(\mathrm{C}=\mathrm{N})$, 165.0, $175.9(2 \mathrm{C}=\mathrm{O}), 61.4\left(\mathrm{CH}_{2}\right), 142.1\left(\mathrm{CH}_{3}\right), 12.2$ (C -19), 23.7 (C-18), 145.3, 83.1 (C=C). MS (EI) m/z (\%): $526\left(\mathrm{M}^{+}, 31 \%\right), 414$ (26), 330 (100), 211 (8.7), 197(1.1). Calcd. for $\mathrm{C}_{29} \mathrm{H}_{42} \mathrm{~N}_{4} \mathrm{O}_{5}$ (526.68): C, 66.14; H, 8.04; N, 10.46\%, Found: C, 66.32; H, $8.21 ; \mathrm{N}, 10.59 \%$.

\subsubsection{Preparation of compounds of compound 11}

To a mixture of compound 1 ( $0.385 \mathrm{~g}, 1 \mathrm{~m} \mathrm{~mol})$ and salicylaldhyde $\mathbf{1 0}(0.122 \mathrm{~g}, 1 \mathrm{~m} \mathrm{~mol})$ in dioxane $(30 \mathrm{ml})$ catalytic amount of piperidine $(0.5 \mathrm{~mL})$ was added. The reaction mixture was heated under reflux for $3 \mathrm{hr}$. until all the reactants had disappeared as indicated by TLC. Leave the mixture until cooling, poured on crushed ice/water mixture, neutralized with hydrochloric acid. The formed solid product was collected by filtration and crystallized from absolute ethanol to form orange crystals of compound 11.

(E)-10,13-dimethyl-17-(2-(2-oxo-2H-chromene-3 carbonyl)hydrazineylidene)hexadecahydro-1Hcyclopenta[a]phenanthren-3-yl acetate (11).

Orange crystals from absolute ethanol, yield $0.47 \mathrm{~g}$ (90\%); mp 210-213 ${ }^{\circ} \mathrm{C}$; IR $\left(\mathrm{KBr}, \mathrm{cm}^{-1}\right):$ v 2926, $2853\left(\mathrm{CH}_{3}, \mathrm{CH}_{2}\right), 1676$ (acetate $\left.\mathrm{C}=\mathrm{O}\right), 1646(\mathrm{C}=\mathrm{N})$, $1608(\mathrm{C}=\mathrm{O}), 1576(\mathrm{C}=\mathrm{C}) .{ }^{1} \mathrm{H}$ NMR (DMSO- $d_{6}$, ppm): $\delta=0.79$ (s, 3H, 19- $\left.\mathrm{CH}_{3}\right), 1.2\left(\mathrm{~s}, 3 \mathrm{H}, 18-\mathrm{CH}_{3}\right)$, $2.50\left(\mathrm{~s}, 3 \mathrm{H}, \mathrm{CH}_{3} \mathrm{CO}\right), 3.79(\mathrm{~m}, 1 \mathrm{H}, \mathrm{C} 5 \alpha \mathrm{H}), 6.58-$ $7.34(\mathrm{~m}, 4 \mathrm{H}$, aromatic $\mathrm{H}), 8.67\left(\mathrm{~s}, 1 \mathrm{H}, \mathrm{NH}, \mathrm{D}_{2} \mathrm{O}-\right.$ exchangeable). ${ }^{13} \mathrm{C}$ NMR (DMSO- $\left.d_{6}, \mathrm{ppm}\right): \delta=37.7$ (C-1), 27.3 (C-2), 73.6 (C-3), 34.6 (C-4), 44.0 (C-5), 25.0 (C-6), 31.1 (C-7), 34.3 (C- 8), 53.7(C-9),36.7 (C-10), 25.7 (C-11), 35.7 (C-12), 39.6 (C-13), 69.8 (C-14), 36.3 (C-15), 31.8 (C-16), 163.1 (C-17), 20.5 (C 18), 21.6 (C-19), 170.3 (CO, acetate), $21.9\left(\mathrm{CH}_{3}-\right.$ acetate), 170.2, $168.7(\mathrm{C}=\mathrm{O}), 166.5(\mathrm{C}=\mathrm{N}), 159.0$ $(\mathrm{C}=\mathrm{C}), \quad 116.0, \quad 118.1, \quad 128.3, \quad 127.9 \quad$ (aromatic carbon). MS (EI) m/z (\%): $518\left(\mathrm{M}^{+}, 33 \%\right), 345$ (16), 330 (25), 189 (22), 173 (100). Calcd. for $\mathrm{C}_{31} \mathrm{H}_{38} \mathrm{~N}_{2} \mathrm{O}_{5}$ (518.65) C, 71.79; H, 7.39; N, 5.40\%, Found: C, 71.65; H, 7.61; N, 5.62\%.

\subsubsection{Preparation of compounds of compounds 14, $17,18,21$ and 22}

A mixture of compound $1(0.385 \mathrm{~g}, 1 \mathrm{~m} \mathrm{~mol})$, potassium hydroxide $(0.05 \mathrm{~g}, 1 \mathrm{~m} \mathrm{~mol})$, and phenylisothiocaynate $(0.11 \mathrm{~g}, 1 \mathrm{~m} \mathrm{~mol})$ in dry $N, N$ dimethylformamide $(10 \mathrm{~mL})$ was stirred for $2 \mathrm{~h}$. The appropriate of phenacyl bromide $(0.2 \mathrm{~g}, 1 \mathrm{~m} \mathrm{~mol})$, chloroacetone $(0.092 \mathrm{~g}, 1 \mathrm{~m} \mathrm{~mol})$, ethylchloroacetate $(0.2 \mathrm{~g}, 1 \mathrm{~m} \mathrm{~mol})$, acetic acid or iodomethane (10 
mmol) was added. The mixture was stirred for 30-60 min. at room temperature until all the reactants had disappeared as indicated by TLC. The mixture, in each case, was poured on crushed ice/water mixture, neutralized with dilute hydrochloric acid. The solid product that formed was collected by filtration, crystallized from absolute ethanol.

(Z)-17-(2-(4-amino-5-benzoyl-2-

(phenylamino)thiophene-3-

carbonyl)hydrazineylidene)-10,13-

dimethylhexadecahydro-1H-

cyclopenta[a]phenanthren-3-yl acetate (14).

Orange crystals from absolute ethanol (20) ml, yield $0.26 \mathrm{~g}$ (39\%); mp 268-270 ${ }^{\circ} \mathrm{C}$; IR $\left(\mathrm{KBr}, \mathrm{cm}^{-1}\right): \mathrm{v}$ $3428\left(\mathrm{NH}_{2}\right), 3060(\mathrm{NH}), 2926-2853\left(\mathrm{CH}_{3}, \mathrm{CH}_{2}\right)$, 1731 (acetate $\mathrm{C}=\mathrm{O}), 1593(\mathrm{C}=\mathrm{C}) .{ }^{1} \mathrm{H}$ NMR (DMSO$\left.d_{6}, \mathrm{ppm}\right): \delta=0.80\left(\mathrm{~s}, 1 \mathrm{H}, 19-\mathrm{CH}_{3}\right), 1.25(\mathrm{~s}, 3 \mathrm{H}, 18-$ $\left.\mathrm{CH}_{3}\right), 2.50\left(\mathrm{~s}, 3 \mathrm{H}, \mathrm{CH}_{3} \mathrm{CO}\right), 3.90(\mathrm{~m}, 1 \mathrm{H}, \mathrm{C} 5-\alpha \mathrm{H})$ $4.60(\mathrm{~m}, 1 \mathrm{H}, \mathrm{CH}-3), 7.23-7.95(\mathrm{~m}, 12 \mathrm{H}, \mathrm{Ar}-\mathrm{H}$ and $\mathrm{NH}_{2}$ ), 9.50 (s, $1 \mathrm{H}, \mathrm{NH}, \mathrm{D}_{2} \mathrm{O}$-exchangeable), 10.21 (s, $1 \mathrm{H}, \mathrm{NH}, \mathrm{D}_{2} \mathrm{O}$-exchangeable). ${ }^{13} \mathrm{C}$ NMR (DMSO$\left.d_{6}, \mathrm{ppm}\right): \delta=37.7$ (C-1), $27.3(\mathrm{C}-2), 73.6$ (C-3), 34.6 (C-4), 44.0 (C-5), 27.0 (C-6), 31.1 (C-7), 34.3 (C-8), 53.7 (C-9), 35.7 (C-10), 21.0 (C-11), 31.1 (C-12), 42.4 (C-13), 63.3 (C-14), 33.3 (C-15), 25.7 (C-16), 166.5 (C-17), 23.7 (C-18), 12.6 (C-19), 21.0 $\left(\mathrm{CH}_{3}\right.$-acetate), $170.3(\mathrm{CO}$, acetate $\left.),\right), 185.9(\mathrm{C}=\mathrm{O})$, $166.5(\mathrm{CN}), 117.8,128.8,129.5,132.6,$, (aromatic carbons). MS (EI) m/z (\%): $666(\mathrm{M}+$, 40\%), 574 (1), 350 (9), 316 (5), 105 (100), 92 (12); Calcd. For $\mathrm{C}_{39} \mathrm{H}_{46} \mathrm{~N}_{4} \mathrm{O}_{4} \mathrm{~S}$ (666.88): C, 70.24; H, 6.95; N, 8.40; S, 4.81\%, Found: C, 70.49; H, 6.78; N, $8.54 ; \mathrm{S}, 4.69 \%$.

(Z)-17-(2-((E)-2-cyano-3-((2-oxopropyl)thio)-3(phenylamino)acryloyl)hydrazineylidene)-10,13dimethylhexadecahydro-1H-

cyclopenta[a]phenanthren-3-yl acetate (17).

Pale brown from absolute ethanol (20) $\mathrm{ml}$, yield $0.31 \mathrm{~g}(51 \%) ; \mathrm{mp} 120-123^{\circ} \mathrm{C}$; IR $\left(\mathrm{KBr}, \mathrm{cm}^{-1}\right): \mathrm{v}$ 3322-3250 (broad peak NH groups), $2927\left(\mathrm{CH}_{3}\right)$, $2850\left(\mathrm{CH}_{2}\right), 2210(\mathrm{CN}), 1597(\mathrm{C}=\mathrm{C}), 1506(\mathrm{C}=\mathrm{N})$. ${ }^{1} \mathrm{H}$ NMR (DMSO- $\left.d_{6}, \mathrm{ppm}\right): \delta=0.80(\mathrm{~s}, 3 \mathrm{H}, 19-$ $\left.\mathrm{CH}_{3}\right), 1.01\left(\mathrm{~s}, 3 \mathrm{H}, 18-\mathrm{CH}_{3}\right), 2.02\left(\mathrm{~s}, 3 \mathrm{H}, \mathrm{OCH}_{3}\right)$, $2.28\left(\mathrm{~s}, 3 \mathrm{H}, \mathrm{CH}_{3} \mathrm{CO}\right), 6.87-7.90\left(\mathrm{~m}, 5 \mathrm{H}, \mathrm{C}_{6} \mathrm{H}_{5}\right)$,
10.07 (s, 1H, NH, $\mathrm{D}_{2} \mathrm{O}$-exchangeable), 12.05 (s, 1H, $\mathrm{NH}, \mathrm{D}_{2} \mathrm{O}$-exchangeable). Ms (EI) m/z (\%): $604\left(\mathrm{M}^{+}\right.$, 37\%). Calcd. for $\mathrm{C}_{34} \mathrm{H}_{44} \mathrm{~N}_{4} \mathrm{O}_{4} \mathrm{~S}$ (604.81) C,67.52; H, 7.33; N, 9.26; S,5.30\%, Found: C, 67.69; H, 7.48; N, 9.37; S, $5.45 \%$.

Ethyl 2-(((E)-3-(2-)((Z)-3-acetoxy-10,13-

dimethylhexadecahydro-17H-

cyclopenta[a]phenanthren-17-

ylidene)hydrazineyl)-2-cyano-3-oxo-1(phenylamino)prop-1-en-1-yl)thio)acetate (18).

Brown crystals from absolute ethanol (20) $\mathrm{ml}$, yield $0.31 \mathrm{~g} \mathrm{(48 \% );} \mathrm{mp} \mathrm{(130-132)}{ }^{\circ} \mathrm{C}$; IR $\left(\mathrm{KBr}, \mathrm{cm}^{-1}\right): \mathrm{v}$ $3404(\mathrm{NH}), 2926\left(\mathrm{CH}_{3}\right), 2858\left(\mathrm{CH}_{2}\right), 1620(\mathrm{C}=\mathrm{N})$, $1592(\mathrm{C}=\mathrm{C}), 1702(\mathrm{C}=\mathrm{O}) .{ }^{1} \mathrm{H}$ NMR (DMSO- $d_{6}$, ppm): $\delta=0.93$ (s, 3H, Me-19), 1.14 (s, 3H, Me-18), 1.25 (t,3H, $\mathrm{CH}_{3}$-ester), 4.18 (q, $\left.2 \mathrm{H}, \mathrm{CH}_{2}\right), 6.87-7.90$ $\left(\mathrm{m}, \quad 5 \mathrm{H}, \quad \mathrm{C}_{6} \mathrm{H}_{5}\right), \quad 10.08 \quad\left(\mathrm{~s}, \quad 1 \mathrm{H}, \quad \mathrm{NH}, \quad \mathrm{D}_{2} \mathrm{O}-\right.$ exchangeable $), \quad 12.01 \quad\left(\mathrm{~s}, \quad 1 \mathrm{H}, \quad \mathrm{NH}, \quad \mathrm{D}_{2} \mathrm{O}-\right.$ exchangeable). MS (EI) m/z (\%) $634\left(\mathrm{M}^{+}, 37 \%\right)$. Calcd. for $\mathrm{C}_{35} \mathrm{H}_{46} \mathrm{~N}_{4} \mathrm{O}_{5} \mathrm{~S}$ (634.84): C, 66.22; H,7.30; $\mathrm{N}, 8.83 ; \mathrm{S}, 5.05 \%$, Found C, 66,41; H, 7.45; N, $8.70 ; \mathrm{S}, 5.19 \%$.

(Z)-17-(2-((E)-2-cyano-3-mercapto-3-

(phenylamino)acryloyl)hydrazineylidene)-10,13-

dimethylhexadecahydro-1H-

cyclopenta[a]phenanthren-3-yl acetate (21).

Orange crystals from absolute ethanol (20) $\mathrm{ml}$ yield $0.35 \mathrm{~g}(63 \%) ; \mathrm{mp} 150-152^{\circ} \mathrm{C}$; IR $\left(\mathrm{KBr}, \mathrm{cm}^{-1}\right)$ : v $3427(\mathrm{NH}), 2927-2854\left(\mathrm{CH}_{3}, \mathrm{CH}_{2}\right), 2174(\mathrm{CN})$, 1730 (acetate $\mathrm{C}=\mathrm{O}), 1625(\mathrm{C}=\mathrm{N}) 1582(\mathrm{C}=\mathrm{C}) .{ }^{1} \mathrm{H}$ NMR (DMSO- $\left.d_{6}, \mathrm{ppm}\right): \delta=0.80\left(\mathrm{~s}, 1 \mathrm{H}, 19 \mathrm{H}-\mathrm{CH}_{3}\right)$, 1.09 (s, 3H, 18- $\left.\mathrm{CH}_{3}\right), 2.03$ (s, 3H, $\left.\mathrm{CH}_{3} \mathrm{CO}\right), 7.12-$ $7.95(\mathrm{~m}, 6 \mathrm{H}$, aromatic hydrogen and 2NH)., 9.75 (s, $1 \mathrm{H}, \mathrm{NH}, \mathrm{D}_{2} \mathrm{O}$-exchangeable). ${ }^{13} \mathrm{C}$ NMR (DMSO- $d_{6}$, ppm): $\delta=39.1$ (C-1), 24.2 (C-2), 41.1 (C-3), 30.0 (C-4), 41.0 (C-5), 27.2 (C-6), 31.2 (C-7), 34.3 (C8), 53.3 (C-9), 35.7 (C-10), 21.0 (C-11), 31.3 (C12), 42.0 (C-13), 63.3 (C-14), 33.9 (C-15), 31.3 (C16), 167.8 (C-17), 23.5 (C-18), 12.2 (C-19), 168.7 $(\mathrm{C}=\mathrm{O}), 166.5(\mathrm{C}=\mathrm{N}), 175.0(\mathrm{C}=\mathrm{O}), 114.5(\mathrm{CN}), 52.2$ $\left(\mathrm{CH}_{3}\right.$-acetate). MS (EI) m/z (\%): $548\left(\mathrm{M}^{+}, 33 \%\right)$, 489 (10), 137(7), 59 (25). Calcd. For $\mathrm{C}_{31} \mathrm{H}_{40} \mathrm{~N}_{4} \mathrm{O}_{3} \mathrm{~S}$ (548.75): C, 67.85; H, 7.35; N, 10.21; S, 5.84\%, Found: C, 67.71; H, 7.47; N, 10.01; S, 5.71\%. 
(Z)-17-(2-((E)-2-cyano-3-(methylthio)-3-

(phenylamino)acryloyl)hydrazineylidene)-10,13-

dimethylhexadecahydro-1H-

cyclopenta[a]phenanthren-3-yl acetate (22).

Orange crystals from absolute ethanol (20) $\mathrm{ml}$, yield $0.28 \mathrm{~g}(49 \%) ; \mathrm{mp} 104-106{ }^{\circ} \mathrm{C}$; IR $\left(\mathrm{KBr}, \mathrm{cm}^{-1}\right)$ : v $3404(\mathrm{NH}), \quad 3053$ (CH-aromatic), $2927-2855$ $\left(\mathrm{CH}_{3}, \mathrm{CH}_{2}\right), 2186(\mathrm{CN}), 1662$ (acetate $\left.\mathrm{C}=\mathrm{O}\right), 1542$ $(\mathrm{C}=\mathrm{C}) .{ }^{1} \mathrm{H}$ NMR (DMSO- $\left.d_{6}, \mathrm{ppm}\right): \delta=0.81(\mathrm{~s}, 1 \mathrm{H}$, $\left.18-\mathrm{CH}_{3}\right), 1.00\left(\mathrm{~s}, 3 \mathrm{H}, 19-\mathrm{CH}_{3}\right), 2.5\left(\mathrm{~s}, 3 \mathrm{H}, \mathrm{SCH}_{3}\right)$, $3.37\left(\mathrm{~s}, 3 \mathrm{H}, \mathrm{OCH}_{3}\right), 4.53$ (s, 1H, C3- $\left.\alpha \mathrm{H}\right), 6.79-7.07$ $(\mathrm{m}, 5 \mathrm{H}$, aromatic hydrogen), $10.74(\mathrm{~s}, 1 \mathrm{H}, \mathrm{NH}$, $\mathrm{D}_{2} \mathrm{O}$-exchangeable $), 12.05\left(\mathrm{~s}, \quad 1 \mathrm{H}, \quad \mathrm{NH}, \quad \mathrm{D}_{2} \mathrm{O}-\right.$ exchangeable). ${ }^{13} \mathrm{C}$ NMR (DMSO- $\left.d_{6}, \mathrm{ppm}\right): \delta=37.7$ (C-1), 27.3 (C-2), 73.6 (C-3), 34.0 (C-4), 44.0 (C-5), 27.2 (C-6), 31.2 (C-7), 34.0 (C-8), 53.2 (C-9), 36.0 (C-10), 21.0 (C-11), 31.2 (C-12), 42.1 (C-13), 63.2 (C-14), 33.0 (C-15), 25.2 (C-16), 166.3 (C-17), 21.0 $\left(\mathrm{CH}_{3}\right), 170(\mathrm{C}=\mathrm{O}), 114.5(\mathrm{CN}), 166.7(\mathrm{C}=\mathrm{O}), 125.4$, 129.5, 126.4, 136.0 (aromatic carbons), 69.1, 178.7 $(\mathrm{C}=\mathrm{C})$. MS (EI) m/z (\%): $562\left(\mathrm{M}^{+}, 34 \%\right), 373(4)$, 346 (22.7), 246 (100), 318 (2.5). Calcd. for $\mathrm{C}_{32} \mathrm{H}_{42} \mathrm{~N}_{4} \mathrm{O}_{3} \mathrm{~S}$ : (562.77) $\mathrm{C}, 68.30 ; \mathrm{H}, 7.52 ; \mathrm{N}, 9.96$; S, $5.70 \%$, Found C, 68.47; H, 7.69; N, 9.73; S, $5.82 \%$.

\subsubsection{Preparation of compounds $23 \& 24$}

To a solution of compound $\mathbf{1 7}$ or $\mathbf{1 8}(1 \mathrm{~m} \mathrm{~mol})$ in absolute ethanol $(20 \mathrm{ml})$, piperdine $(1 \mathrm{ml})$ was added. The reaction mixture, in each case, was heated under reflux for $2-3 \mathrm{hr}$, until all the reactants had disappeared as indicated by TLC. Leave the mixture until cooling, poured on crushed ice/water mixture, neutralized with dilute hydrochloric acid. The solid product that formed was collected by filtration, crystallized from absolute ethanol.

(Z)-17-(2-(5-acetyl-4-amino-2-

(phenylamino)thiophene-3-

carbonyl)hydrazineylidene)-10,13-

dimethylhexadecahydro-1H-

cyclopenta[a]phenanthren-3-yl acetate (23).

Brown crystals from absolute ethanol (20) ml, yield $0.43 \mathrm{~g}(71 \%)$; mp $125-127^{\circ} \mathrm{C}$; IR $\left(\mathrm{KBr}, \mathrm{cm}^{-1}\right)$ : v $3428\left(\mathrm{NH}_{2}\right), 3371(\mathrm{NH}), 2925-2853\left(\mathrm{CH}_{3}, \mathrm{CH}_{2}\right)$, 1731 (acetate $\mathrm{C}=\mathrm{O}) 1574(\mathrm{C}=\mathrm{C}) .{ }^{1} \mathrm{H}$ NMR (DMSO- $\left.d_{6}, \mathrm{ppm}\right) \delta=0.80\left(\mathrm{~s}, 1 \mathrm{H}, 19-\mathrm{CH}_{3}\right), 1.12(\mathrm{~s}, 3 \mathrm{H}, 18-$ $\left.\mathrm{CH}_{3}\right), 2.02$ (s, 3H, $\left.\mathrm{CH}_{3} \mathrm{CO}\right), 4.57$ (m, $\left.1 \mathrm{H}, \mathrm{CH}-3\right)$, 6.89-7.57 (m, 5H, aromatic $\mathrm{H}), 6.01\left(\mathrm{~s}, 2 \mathrm{H}, \mathrm{NH}_{2}\right.$, $\mathrm{D}_{2} \mathrm{O}$-exchangeable $), \quad 9.85 \quad\left(\mathrm{~s}, \quad 1 \mathrm{H}, \quad \mathrm{NH}, \quad \mathrm{D}_{2} \mathrm{O}-\right.$ exchangeable $), \quad 10.45 \quad\left(s, \quad 1 \mathrm{H}, \quad \mathrm{NH}, \quad \mathrm{D}_{2} \mathrm{O}-\right.$ exchangeable). ${ }^{13} \mathrm{C}$ NMR (DMSO- $d_{6}, \mathrm{ppm}$ ): $\delta=37.7$ (C-1), 27.3 (C-2), 73.6 (C-3), 34.6 (C-4), 44.2 (C5), 27.8 (C-6), 31.1 (C-7), 34.3 (C-8), 53.7 (C-9), 35.7 (C-10), 21.0 (C-11), 31.1 (C-12), 42.4 (C-13), 63.3 (C-14), 33.3 (C-15), 25.7 (C-16), 166.5 (C-17), 23.7 (C-18), 12.6 (C-19), $21.1\left(\mathrm{CH}_{3}\right), 170.3(\mathrm{CO}$, acetate), 21.0 ( $\mathrm{CH}_{3}$-acetate $), 163.9$ (CO), 166.5 $(\mathrm{CN}), 117.8,122.4,129.5,139.6$ (aromatic carbons). Ms (EI) m/z (\%): $604\left(\mathrm{M}^{++}, 6.6 \%\right), 472$ (30), 316 (1), 231 (2). Calcd. for $\mathrm{C}_{34} \mathrm{H}_{44} \mathrm{~N}_{4} \mathrm{O}_{4} \mathrm{~S}$ (604.81) C, 67.52; H, 7.33; N, 9.26; S, 5.30\%, Found: C, 67.73; H, 7.48; N, 9.42; S, 5.51\%.

Ethyl(Z)-4-(2-(3-acetoxy-10,13-

dimethylhexadecahydro-17H-

cyclopenta[a]phenanthren-17-ylidene)hydrazine-1carbonyl)-3-amino-5-(phenylamino)thiophene-2carboxylate (24).

Pale brown crystals from absolute ethanol (20) $\mathrm{ml}$, yield $0.24 \mathrm{~g}(38 \%)$; $\mathrm{mp}(133-135)^{\circ} \mathrm{C}$; IR $\left(\mathrm{KBr}, \mathrm{cm}^{-}\right.$ $\left.{ }^{1}\right)$ : v 3368, 3200, $3105\left(\mathrm{NH}_{2}, \mathrm{NH}\right), 2928,2854$ $\left(\mathrm{CH}_{3}, \mathrm{CH}_{2}\right), 1702(\mathrm{C}=\mathrm{O}) .{ }^{1} \mathrm{H}$ NMR (DMSO- $d_{6}$, ppm): $\delta=0.87\left(\mathrm{~s}, 1 \mathrm{H}, 19-\mathrm{CH}_{3}\right), 0.98(\mathrm{~S}, 1 \mathrm{H}, 18-$ $\mathrm{CH}_{3}$ ), 1.34 (s, 3H, $\mathrm{CH}_{3}$-ester), 2.03 (s, 3H, $\mathrm{CH} 3 \mathrm{CO}$ ), 3.76 (s $, 3 \mathrm{H}, \mathrm{CH}_{3}$, acetate), 4.29 (q, $5 \mathrm{H}, \mathrm{CH}_{2} \mathrm{CH}_{3}$ ), 6.89-7.67 (m, 6H, aromatic $\mathrm{H}), 8.41\left(\mathrm{~s}, 2 \mathrm{H}, \mathrm{NH}_{2}\right.$, $\mathrm{D}_{2} \mathrm{O}$-exchangeable $), 10.00 \quad\left(\mathrm{~s}, \quad 1 \mathrm{H}, \quad \mathrm{NH}, \quad \mathrm{D}_{2} \mathrm{O}-\right.$ exchangeable), $10.50 \quad(\mathrm{~s}, 1 \mathrm{H}, \mathrm{NH}),{ }^{13} \mathrm{C} \quad \mathrm{NMR}$ (DMSO- $d 6, \mathrm{ppm}$ ): $\delta=39.2$ (C-1), 24.3 (C-2) 41.6 (C-3), 30.6 (C-4), 41.2 (C-5), 27.0 (C-6), 31.1 (C-7), 34.0 (C-8), 53.7 (C-9), 36.7 (C-10), 21.0 (C-11), 31.1 (C-12), 42.4 (C-13), 63.3 (C-14), 33.3 (C-15), 25.7 (C-16), 166.0 (C-17), 23.7 (C-18), 12.6 (C-19), $14.1\left(\mathrm{CH}_{3}\right), 60.1\left(\mathrm{CH}_{2}\right), 175.3(\mathrm{CO}$, acetate $), 21.0$ $\left(\mathrm{CH}_{3}\right.$-acetate), $160.9(\mathrm{CO}), 166.5(\mathrm{CN}), \quad 118.0$, 120.3, 120.3, 167,2 (CH, thiophene), 117.8, 122.4, 129.5, 139.9 (aromatic carbons). MS (EI) m/z (\%) $634\left(\mathrm{M}^{+}, 50 \%\right), 546$ (5), 371 (48), 382 (64). Calc for $\mathrm{C}_{35} \mathrm{H}_{46} \mathrm{~N}_{4} \mathrm{O}_{5} \mathrm{~S}$ (634.84): C,66.22; H, 7.30; N, 8.83; S, 
$5.05 \%$, Found C, 66,39; H, 7.45; N, 8.71; S, 4.87 $\%$.

\subsubsection{Preparation of compound26}

To a mixture of compound $\mathbf{1}(0.385 \mathrm{~g}, 1 \mathrm{~m} \mathrm{~mol})$, phenylisothiocynate $(0.11 \mathrm{~g}, 1 \mathrm{~m} \mathrm{~mol})$ and sulphur $(0.33 \mathrm{~g}, 1 \mathrm{~m} \mathrm{~mol})$ in dioxane $(30 \mathrm{ml})$, few drops TEA were added. The reaction mixture was heated under reflux for 3 hrs. leave the mixture until cooling, poured on crushed ice/water mixture and then neutralized with hydrochloric acid. The solid product that formed was collected by filtration, crystallized from absolute ethanol to give brown crystals of compound $\mathbf{2 6}$.

(Z)-17-(2-(4-amino-3-phenyl-2-thioxo-2,3dihydrothiazole-5-carbonyl)hydrazineylidene)-

10,13-dimethylhexadecahydro-1Hcyclopenta[a]phenanthren-3-yl acetate (26).

Brown crystals from absolute ethanol yield $0.36 \mathrm{~g}$ (62\%); mp 218-220 ${ }^{\circ}$; IR ( $\left.\mathrm{KBr}, \mathrm{cm}^{-1}\right):$ v $3750(\mathrm{NH}$, $\left.\mathrm{NH}_{2}\right), 2853-2367\left(\mathrm{CH}_{3}, \mathrm{CH}_{2}\right), 2075(\mathrm{C}=\mathrm{O}), 1632$ $(\mathrm{C}=\mathrm{N}), 1496(\mathrm{C}=\mathrm{C}) .{ }^{1} \mathrm{HNMR}$ (DMSO- $\left.d_{6}, \mathrm{ppm}\right): \delta=$ 0.99 (s, 3H, 19- $\left.\mathrm{CH}_{3}\right), 1.25$ (s, 3H, 18- $\left.\mathrm{CH}_{3}\right), 2.03$ (s, $\left.3 \mathrm{H}, \mathrm{CH}_{3} \mathrm{CO}\right), 4.41$ (m, 1H, CH-4), 6.25 (s, 2H, $\mathrm{NH}_{2}-$ $\mathrm{D}_{2} \mathrm{O}$-exchangeable $), 7.22-7.65(\mathrm{~m}, 5 \mathrm{H}$, aromatic hydrogen ), 8.90 (s, H, NH-D $\mathrm{D}_{2} \mathrm{O}$-exchangeable). ${ }^{13} \mathrm{C}$ NMR (DMSO-d6, ppm): $\delta=38.7$ (C-1), 24.5 (C-2), 41.1 (C-3), 30.1 (C-4), 42.6 (C-5), 27.0 (C-6), 31.1 (C-7), 34.2 (C-8), 53.0 (C-9), 35.5 (C-10), 21.0 (C11), 31.0 (C-12), 36.2 (C-13), 63.3 (C-14), 33.9 (C15), $31.7(\mathrm{C}-16), 168.3(\mathrm{C}=\mathrm{N}), 128.4,129.0,126.4$ (aromatic carbons), $12.2(\mathrm{C}-\mathrm{CH}), 23.7\left(\mathrm{C}-\mathrm{CH}_{3}-18\right)$, 184.4 (C=S), 75.9 (CS). MS (EI) m/z (\%): $580\left(\mathrm{M}^{+}\right.$, 33\%), 374 (23), 316 (100), 266 (9.0), 275 (2.7). Calcd. for $\mathrm{C}_{31} \mathrm{H}_{48} \mathrm{~N}_{4} \mathrm{O}_{3} \mathrm{~S}_{2}$ (580.81): $\mathrm{C}, 64.11 ; \mathrm{H}$, 6.94; N, 9.65; S, 11.04\%, Found: C, 64.34; H, 6.68; $\mathrm{N}, 9.97 ; \mathrm{S}, 11.13 \%$.

amount of progesterone $(1.57 \mathrm{~g}, 0.005 \mathrm{~mol})$ and thiose-

micarbazide $(0.45 \mathrm{~g}, 0.005 \mathrm{~mol})$ in ethanol $(20 \mathrm{~mL})$ containing $\mathrm{HCl}$

$(1 \mathrm{~mL})$ was heated under reflux for $1 \mathrm{~h}$. The reaction mixture was

poured onto ice/water and the solid product collected by filtration and crystallized from absolute ethanol

\subsubsection{Preparation of compound 29}

A mixture of acetyl epi-androsterone $(0.385 \mathrm{~g}, 1 \mathrm{~m}$ $\mathrm{mol})$, and thiosemicarbazide $(0.09 \mathrm{~g}, 1 \mathrm{~m} \mathrm{~mol})$ in absolute ethanol $(20 \mathrm{~mL})$ containing $\mathrm{HCl}(0.5 \mathrm{~mL})$ was heated under reflux for $1 \mathrm{~h}$. The reaction mixture was poured onto ice/water and the solid product collected by filtration and crystallized from absolute ethanol.

(Z)-17-(2-carbamothioylhydrazineylidene)-10,13-

\section{dimethylhexadecahydro-1H-}

cyclopenta[a]phenanthren-3-yl acetate (29).

Pale yellow crystals from absolute ethanol, yield $0.335 \mathrm{~g}$ (82\%); mp 233-235 ${ }^{\circ} \mathrm{C}$; IR $\left(\mathrm{KBr}, \mathrm{cm}^{-1}\right): \mathrm{V}$ 3421-3216 ( $\left.\mathrm{NH}_{2}, \mathrm{NH}\right), 2930,2863\left(\mathrm{CH}_{3}, \mathrm{CH}_{2}\right)$, $1728(\mathrm{C}=\mathrm{O}), 1644(\mathrm{C}=\mathrm{N}), 1525(\mathrm{C}=\mathrm{C}), 1171(\mathrm{C}=\mathrm{S})$. ${ }^{1} \mathrm{H}$ NMR (DMSO-d6, ppm): $\delta=0.80$ (s, 3H CH $\left.3-19\right)$, 1.16 (s, 3H, $\left.\mathrm{CH}_{3}-18\right), 2.53$ (s, 3H, $\mathrm{CH}_{3} \mathrm{CO}$ ), 4.61 (m, $1 \mathrm{H}, \mathrm{CH}-\mathrm{C} 3), 7.75$ (s, 2H, $\mathrm{NH}_{2}, \mathrm{D}_{2} \mathrm{O}$-exchangeable), 11.29 (s, 1H, NH, $\mathrm{D}_{2} \mathrm{O}$-exchangeable). (MS (EI) m/z (\%): $405\left(\mathrm{M}^{+}, 23 \%\right)$. Calcd. for $\mathrm{C}_{22} \mathrm{H}_{35} \mathrm{~N}_{3} \mathrm{O}_{2} \mathrm{~S}$ (405.60): C, 65.15; H, 8.70; N, 10.36; S, $7.90 \%$, Found: C, 65.29; H, 8.56; N, 10.22; S,7.68 \%.

\subsubsection{Preparation of compounds 31,32 and 33}

A mixture of compound $29(0.3 \mathrm{~g}, 1 \mathrm{~m} \mathrm{~mol})$, and the appropriate hydrozonyl halids $(1 \mathrm{~m} \mathrm{~mol})$, in absolute ethanol (30 mL) was refluxed for $3-5 \mathrm{~h}$ in the presence of TEA $(0.5 \mathrm{ml})$. Leave the mixture until cooling, poured on crushed ice/water mixture, neutralized with hydrochloric acid. The products so formed were filtered, washed by cold water. The solid products obtained, in each case, upon cooling were collected by filtration and crystallized from ethanol.

(E)-10,13-dimethyl-17-(((Z)-5-methyl-4- $((E)-$

phenyldiazenyl)thiazol-2(3H)-

ylidene)hydrazineylidene)hexadecahydro-1Hcyclopenta[a]phenanthren-3-yl acetate (31).

Yellow crystals from absolute ethanol (20) ml, yield $0.27 \mathrm{~g}$ (49\%); mp $177-179^{\circ} \mathrm{C}$; IR $\left(\mathrm{KBr}, \mathrm{cm}^{-1}\right): \mathrm{v}$ $3421(\mathrm{NH}), 2930-2862\left(\mathrm{CH}_{3}, \mathrm{CH}_{2}\right), 1730$ (acetate $\mathrm{C}=\mathrm{O}), 1644(\mathrm{C}=\mathrm{N}), 1525(\mathrm{C}=\mathrm{C}) .{ }^{1} \mathrm{H}$ NMR (DMSO$\left.d_{6}, \mathrm{ppm}\right): \delta=0.90\left(\mathrm{~s}, 3 \mathrm{H}, 19-\mathrm{CH}_{3}\right), 1.23$ (s, 3H, 18- 
$\left.\mathrm{CH}_{3}\right), 2.53$ (s, 3H, $\left.\mathrm{CH}_{3} \mathrm{CO}\right), 4.41(\mathrm{~m}, 1 \mathrm{H}, \mathrm{CH}-3)$, 6.96-7.32 (m, 5H, $\left.\mathrm{C}_{6} \mathrm{H}_{5}\right), 10.41\left(\mathrm{~s}, 1 \mathrm{H}, \mathrm{NH}, \mathrm{D}_{2} \mathrm{O}-\right.$ exchangeable).${ }^{13} \mathrm{C}$ NMR (DMSO- $d 6, \mathrm{ppm}$ ): $\delta=39.0$ (C-1), 23.7 (C-2), 40.1 (C-3), 30.1 (C-4), 40.0 (C-5), 27.0 (C-6), 31.1 (C-7), 39.1 (C-8), 35.7 (C-9), 21.7 (C-10), 31.1 (C-11), 42.7 (C-12), 63.3 (C-13), 33.1 (C-14), 120.3, 128.7, 147.9 (aromatic carbons), $175.5(\mathrm{C}=\mathrm{O}), 102.0,138.0(\mathrm{C}=\mathrm{C}), 12.2\left(\mathrm{C}-\mathrm{CH}_{3}-19\right)$, $23.2\left(\mathrm{CH}_{3}\right)$. MS (EI) m/z (\%): $547\left(\mathrm{M}^{+}, 4 \%\right), 545$ (1), 442 (2), 105 (40). Calcd. for $\mathrm{C}_{31} \mathrm{H}_{41} \mathrm{~N}_{5} \mathrm{O}_{2} \mathrm{~S}$ (547.76): C, 67.97; H, 7.54; N, 12.79; S, 5.85\%, Found: C, 67.83; H, 7.40; N, 12.52; S, 5.69\%.

(E)-10,13-dimethyl-17-(((Z)-5-phenyl-4- $((E)-$ phenyldiazenyl)thiazol-2(3H)-

ylidene)hydrazineylidene)hexadecahydro-1Hcyclopenta[a]phenanthren-3-yl acetate (32).

Orange crystals from absolute ethanol (20) $\mathrm{ml}$ yield 0.37g (61\%); mp143-145 ${ }^{\circ} \mathrm{C}$; IR $\left(\mathrm{KBr}, \mathrm{cm}^{-1}\right)$ : v $3435(\mathrm{NH}), 2926-2855\left(\mathrm{CH}_{3}, \mathrm{CH}_{2}\right), 1725$ (acetate $\mathrm{C}=\mathrm{O}), 1643(\mathrm{C}=\mathrm{N}), 1593(\mathrm{C}=\mathrm{C})$; ${ }^{1} \mathrm{H}$ NMR (DMSO$\left.d_{6}, \mathrm{ppm}\right): \delta=0.79\left(\mathrm{~s}, 3 \mathrm{H}, 19-\mathrm{CH}_{3}\right), 1.24$ (s, 3H, 18$\left.\mathrm{CH}_{3}\right), 2.50$ (s, 3H, $\left.\mathrm{CH}_{3} \mathrm{CO}\right), 4.40(\mathrm{~m}, 1 \mathrm{H}, \mathrm{CH}-\mathrm{C} 3)$, 7.32-8.30 (m, 5H, $\left.\mathrm{C}_{6} \mathrm{H}_{5}\right), 9.87\left(\mathrm{~s}, 1 \mathrm{H}, \mathrm{NH}, \mathrm{D}_{2} \mathrm{O}-\right.$ exchangeable). ${ }^{13} \mathrm{C}$ NMR (DMSO- $\left.d_{6}, \mathrm{ppm}\right): \delta=37.3$ (C-1) , 27.3 (C-2), 73.6 (C-3), 46.6 (C-4), 44.6 (C5), 27.0 (C-6) 31.1 (C-7), 34.3 (C-8), 53.7 (C-9), 35.7 (C-10), 21.0 (C-11), 31.0 (C-12), 42.9 (C-13), $63.3(\mathrm{C}-14), 33.9$ (C-15), $26.2(\mathrm{C}-16), 184.3(\mathrm{C}=\mathrm{N})$, 120.8, 128.3, 134.2 (aromatic carbons). (MS (EI) m/z (\%): $609\left(\mathrm{M}^{+}, 10 \%\right), 567$ (60), 490 (10), 330 (1.0). Calcd. for $\mathrm{C}_{36} \mathrm{H}_{43} \mathrm{~N}_{5} \mathrm{O}_{2} \mathrm{~S}$ (609.83): C, 70.90; H,7.11; N, 11.48; S, $5.26 \%$, Found: C, 70.77; $\mathrm{H}, 7.23$; N, 11.30; S, $5.41 \%$.

(E)-10,13-dimethyl-17-(((Z)-5-(naphthalen-1-yl)-4((E)-phenyldiazenyl)thiazol-2(3H)ylidene)hydrazineylidene)hexadecahydro-1Hcyclopenta[a]phenanthren-3-yl acetate (33).

Brown crystals from absolute ethanol (20) ml yield $0.35 \mathrm{~g}(53 \%), \mathrm{mp} 180-183^{\circ} \mathrm{C}$; IR $\left(\mathrm{KBr}, \mathrm{cm}^{-1}\right): \mathrm{V}$ $3438(\mathrm{NH}), 2925-2856\left(\mathrm{CH}_{3}, \mathrm{CH}_{2}\right), 1628$ (acetate $\mathrm{C}=\mathrm{O}))$; ${ }^{1} \mathrm{H}$ NMR (DMSO- $\left.d_{6}, \mathrm{ppm}\right): \delta=0.79(\mathrm{~s}, 3 \mathrm{H}$, 19- $\left.\mathrm{CH}_{3}\right), 1.19\left(\mathrm{~s}, 3 \mathrm{H}, \quad 18-\mathrm{CH}_{3}\right), 2.50(\mathrm{~s}, 3 \mathrm{H}$, $\mathrm{CH}_{3} \mathrm{CO}$ ), 4.40 (m, 1H, CH-C3), 7.64-8.68 (m, 12H, aromatic $\mathrm{H}), 9.77$ (s, 1H, NH, $\mathrm{D}_{2} \mathrm{O}$-exchangeable),
${ }^{13} \mathrm{C}$ NMR (DMSO- $\left.d_{6}, \mathrm{ppm}\right): \delta=39.1(\mathrm{C}-1), 24.7(\mathrm{C}-$ 2), 41.1 (C-3), 30.0 (C-4), 41.0 (C-5), 27.2 (C-6), 31.10 (C-7), 34.30 (C-8), 53.10 (C-9), 21.0 (C-10), 31.00 (C-11), 42.9 (C-12), 63.0 (C-13), 31.1(C-14), 120.3, 128.7, 147.9 (aromatic carbons), 175.9 $(\mathrm{C}=\mathrm{O}), 184.5(\mathrm{C}=\mathrm{N}), 12.2\left(\mathrm{C}-\mathrm{CH}_{3}-19\right), 52.2\left(\mathrm{CH}_{3}\right)$. (MS (EI) m/z (\%) $659\left(\mathrm{M}^{+}, 43 \%\right), 633$ (7), 91 (35), 523 (10), 445 (100) 416 (25). Calcd. for $\mathrm{C}_{40} \mathrm{H}_{45} \mathrm{~N}_{5} \mathrm{O}_{2} \mathrm{~S}$ (659.88): C,72.81; H, 6.87; N, 10.61; S, $4.86 \%$. Found: C, 72.68; H, 7.03; N, 10.41; S, $5.05 \%$.

\subsection{Pharmacological Assay}

\subsubsection{Rats}

Sprague-Daweley strain rats weighing 120-130g or Swiss albino mice $20-25 \mathrm{~g}$ body weight were used throughout the experiments, supplied by the Animal House Colony of the National Research Centre, Cairo, Egypt and maintained under specific pathogen-free conditions on a 12-hour light/dark cycle, with temperature $25 \pm 1^{\circ} \mathrm{Cand}$ humidity $55 \%$. Rats had free access to standard laboratory chow and tap water. Rats procedures were conducted according to the regulations of the Institutional Ethics Committee of the National Research Centre, Giza, Egypt, and in accordance with the recommendations for the proper care and use of laboratory animals [NIH 1985].

\subsubsection{Tests of Inflammation: carrageenan- induced paw oedema assay}

Paw oedema was induced by sub-plantar injection of $100 \mu \mathrm{L}$ of $1 \%$ sterile carrageenan lambda in saline into the right hind paw of rats [Winter et al 1962]. Contralateral paw received an equal volume of saline. Paw volume was determined immediately before carrageenan injection and at selected times thereafter using a plethysmometer (UgoBasile, Milan, Italy). The oedema component of inflammation was quantified by measuring the paw volume $(\mathrm{mL})$ at zero time (before carrageenan injection) and at 1, 2, 3 and $4 \mathrm{~h}$ after carrageenan injection and comparing it with the pre-injection value for each rat. Oedema was expressed as a percentage of change from control (pre-drug, zero 
time) values. The effect of systemic administration of inflammation by sub plantar carrageenan was studied. Rats were allocated into 22 different groups (6 rats/group), Ten tested compounds administrated subcutaneously (s.c.), in two doses (25 or 50 $\mathrm{mg} / \mathrm{kg}$ ), $30 \mathrm{~min}$ before sub-plantar carrageenan. Tested compounds administrated in saline $(0.9 \%$ $\mathrm{NaCl}$ ) with one drop of Tween 80 as a vehicle in all pharmacological studies. The control group of carrageenan-treated rats received an equal volume of saline $30 \mathrm{~min}$ before subplantar carrageenan injection. Another group- administered indomethacin (10 mg/kg,p.o.) served as control positive.

\subsubsection{Gastric ulcerogenic study}

The effect of the different compounds on the development of gastric mucosal damage due to ethanol was studied. Rats were randomly assigned to 22 different treatment groups (6 rats/group). Rats were fasted for $18 \mathrm{~h}$ but allowed water ad libitum. They were orally administered either saline (control) or each one of the test compounds (10 compounds) $30 \mathrm{~min}$ prior to ethanol $(96 \%, 1 \mathrm{~mL}$, p.o.) in two doses $(25$ or $50 \mathrm{mg} / \mathrm{kg}$ ). Rats were killed $1 \mathrm{~h}$ after ethanol administration, their stomachs excised, opened along the greater curvature, and rinsed with saline. Stomachs were then extended on a plastic board and examined for the presence of gastric mucosal lesions. The number and severity of mucosal lesions were noted and lesions were scaled as described by [ Mózsik et al., 1982].

\subsubsection{Statistical analysis}

Data were expressed as mean \pm SE. Data were analyzed by one-way analysis of variance, followed by a Tukey's multiple range tests for post hoc comparison of group means. Effects with a probability of $\mathrm{P}<0.05$ were considered to be significant.

\section{Results and Discussion}

\subsection{Chemistry}

In the communication from the laboratory of steroidal heterocyclic synthesis we have reported about the synthesis, of the cyanoacetylhydrazonoandrostane (1) by the reaction of cyanoacetylhydrazine with an equimolar amount of acetyl epi-androsterone in the presence of acetic acid [Doss et al.,1999]. The pathways of preparing our new hybrid derivatives were assumed up in Schemes $1-5 . \quad$ When cyanoacetylhydrazonoandrostane (1) reacted with sulfur and ethyl cyanoacetate or malononitrile under reflux for 3-5 hrs gave the corresponding thiophenyl hydrazonoandrostane derivatives $\mathbf{4}$ and $\mathbf{5}$ respectively (Scheme1). The structures were confirmed with the analytical and spectral data (c.f. Materials and Method.).

However, when compound (1) reacted with malononitrile or ethyl cyanoacetate in the presence of triethyamine (TEA) under reflux it offered the corresponding oxopyridinyl androstane derivatives $\mathbf{8}$ and 9 respectively. The IR spectra of compounds 8 and 9 showed a strong absorption band with two spikes due to presence of $\mathrm{NH}_{2}$ group, the ${ }^{1} \mathrm{H} \mathrm{NMR}$, ${ }^{13} \mathrm{C}$ NMR and EIMS data of these products are consistent with the proposed structures (c.f. Materials and Method.). Meanwhile, the reaction of compound (1) with saliclylaldhyde in the presence of piperidine gave the chromene hydrazineylidene androstane derivative 11 (Scheme 2). The ${ }^{1} \mathrm{H}$ NMR of compound $\mathbf{1 1}$ showed the protons of chromene moiety which hasn't existed in case of the start, the IR, ${ }^{13} \mathrm{C}$ NMR and EIMS data of compounds are consistent with the proposed structures (c.f. Materials and Method.).

Compound (1) reacted with phenylisothiocynate in dry $N, N$-dimethylformamide for $2 \mathrm{~h}$. and the obtained intermediate was reacted with an appropriate amount of phenacyl bromide, chloroacetone, ethyl chloroacetate, acetic acid or methyl iodide to give compounds 14, 17, 18, 21 and 22 respectively. When compounds 17, 18 were treated with piperiedine in absolute ethanol they gave the corresponding thiophenyl hydrazineylidene androstane derivatives $\mathbf{2 3}$ and $\mathbf{2 4}$ respectively (Scheme 3).

In the same manner, when compound (1) reacted with phenylisothiocynate and sulphur in the 
presence of TEA afforded thiazolyl
hydrazineylidene androstane $\mathbf{2 6}$ (Scheme 4). On the other hand, acetyl epi-androsterone 27 reacted with an equimolar amount of thiosemicarbazides under reflux in absolute ethanol/ $\mathrm{Hcl}$ solution to give the thiosemicarbazonoandrostane derivative 29. The reaction of compound $\mathbf{2 9}$ with hydrozonyl halide derivatives namely acetylhydrozonyl halide 30a, benzooylehydrozonylhalide

30b or naphthoylhydrozonylhalide 30c in the presence of TEA afforded the phenyldiazienethiazolyl hydrazineylidene androstane derivatives $\mathbf{3 1}, \mathbf{3 2}$ and 33 in good yield (Scheme 5). The structures of all products were proved by the analytical and spectral data (c.f. Materials and Method.).

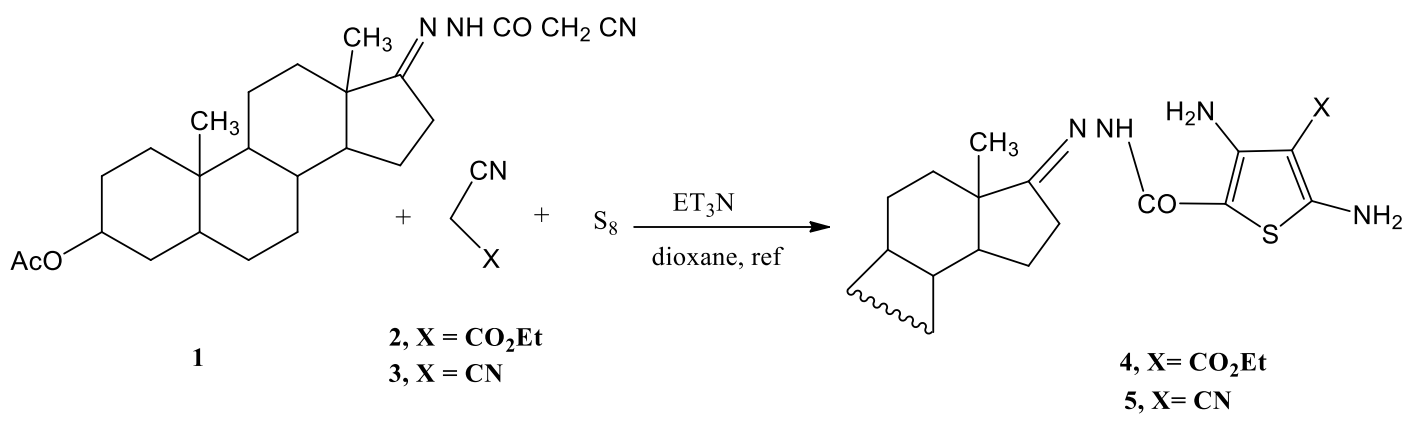

Scheme 1<smiles>CC12CCC3CCC3C1CCC2=N</smiles>

1

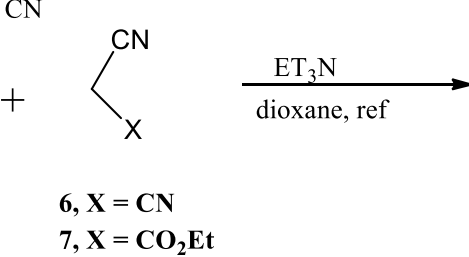<smiles></smiles>

$8, \mathrm{X}=\mathrm{CN}$

9, $\mathrm{X}=\mathrm{CO}_{2} \mathrm{Et}$<smiles>CC(=O)NN=C1CCC2C3CCC3CCC12C</smiles><smiles>O=Cc1ccccc1O</smiles>

10<smiles>CC(C)C1CCC2(C)C(=NNC(=O)c3cc4ccccc4oc3=O)CCC12</smiles>

11 
<smiles>CC12CCC3CCC3C1CCC2=NNC(=O)C#N</smiles>

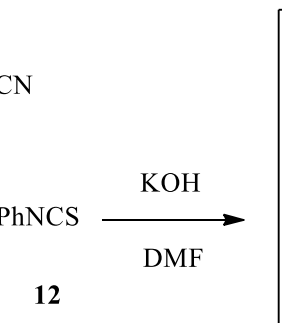<smiles>CC1CCC2(C)C(=NNC(=O)OC(C#N)=C(N)P)CCC2C1C</smiles><smiles>[X]C(Nc1ccccc1)=C(C#N)OCNN=C1CCC2C(CC)CCC12</smiles>

21, $\mathrm{X}=\mathrm{H}$ 22, $\mathrm{X}=\mathrm{CH}_{3}$

<smiles>CC12CCC3CCC3C1CCC2=NCOc1c(Nc2ccccc2)sc(C(=O)Oc2ccccc2)c1N</smiles>

14



$18, X=\mathrm{OC}_{2} \mathrm{H}_{5}$

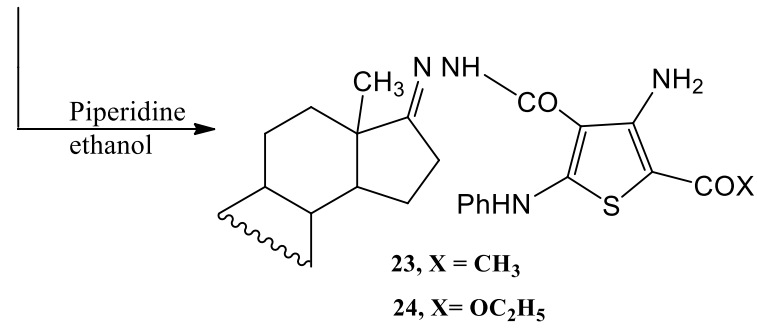

Scheme 3
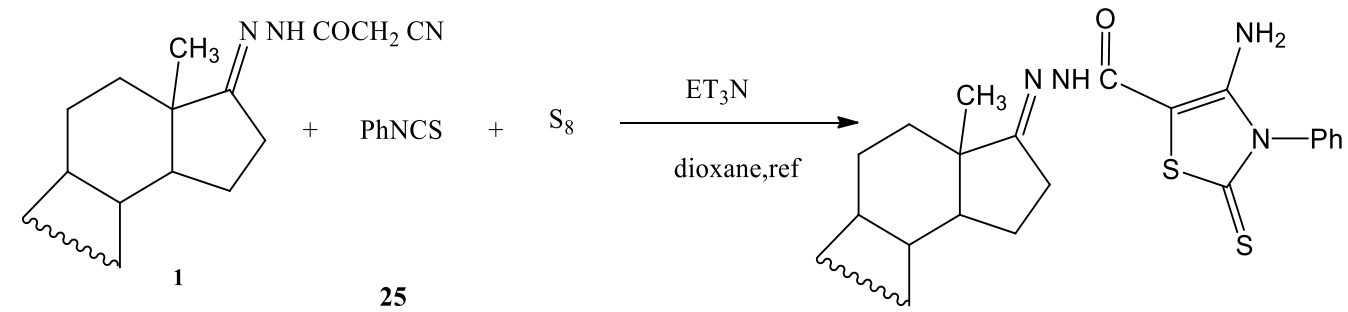

Scheme 4 


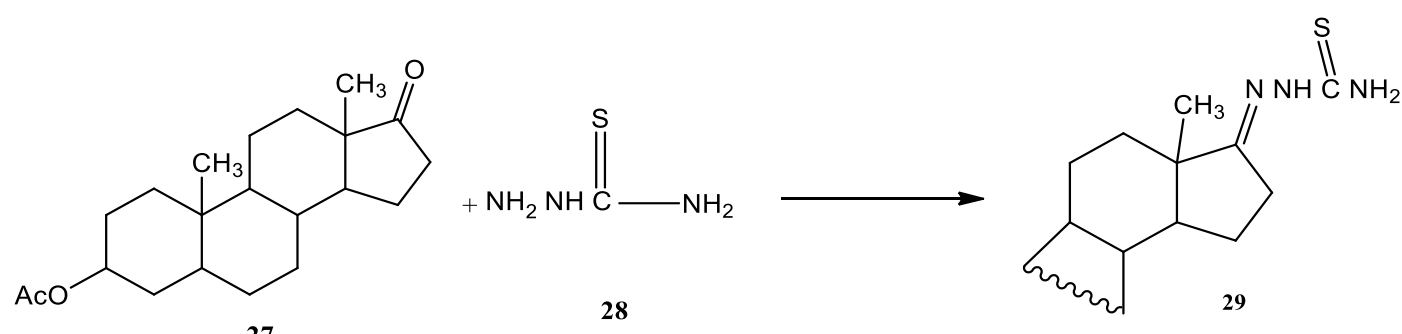

27

28

3.2.



Scheme 5

\section{Pharmacology}

\subsubsection{Anti-inflammatory effects}

The impacts of systemic injection of the observed compounds on the creation of oedema were explored using an inflammation of carrageenan caused paw. Each compound was injected subcutaneously (s.c.), in two doses (25 or 50 $\mathrm{mg} / \mathrm{kg}$ ), $30 \mathrm{~min}$ before sub-plantar carrageenan. Compared to the control group (pre-drug), all evaluated compounds reduced dose-dependent paw oedema (Table 1). Indomethacin was administered as a positive control at $18 \mathrm{mg} / \mathrm{kg}$, s.c., $30 \mathrm{~min}$ before carrageenan. Data are expressed as mean \pm S.E., $\mathrm{n}=6$ per group. The values in parenthesis in Table 1 indicate the percentage (\%) of increase in paw volume (oedema) from basal (zero time) values. The anti-inflammatory drug indomethacin used as a control drug in the study at the dose of $10 \mathrm{mg} / \mathrm{kg}$ inhibited the oedema response to carrageenan at $1,2,3,4$ h time points by $-57.3 \%, 54.1 \%, 61.9 \%$, $60.8 \%$ (Figs 1-5).

Effects of compounds $\mathbf{4}$ and $\mathbf{5}$, the oedema response decreased at 2,3 and 4 h time points postcarrageenan by compound 4 at $25 \mathrm{mg} / \mathrm{kg}$. The percent decrease in oedema formation for the 25 $\mathrm{mg} / \mathrm{kg}$ dose was $-51.7 \%,-51.8 \%,-48.6 \%$. The higher dose resulted in inhibition of oedema formation at all time points in the study by $-60.9 \%$, $62.4 \%,-51.8 \%,-57.1 \%$, Compound 5 given at 25 $\mathrm{mg} / \mathrm{kg}$ inhibited the paw oedema response compared with the control group at $4 \mathrm{~h}$ post-carrageenan by $30.1 \%$ while the higher dose inhibited oedema at $3 \mathrm{~h}$ and $4 \mathrm{~h}$ post-carrageenan by $-30.3 \%$ and $-31.5 \%$ (Fig.1).

Effects of compounds $\mathbf{8}$ and $\mathbf{9}$, Compound $\mathbf{8}$ given at 25 or $50 \mathrm{mg} / \mathrm{kg}$ produced significant inhibition of the oedema response at all time point in the study. The percent inhibition was $-62.5 \%,-67.9 \%,-65 \%$, $63.1 \%$ for the $25 \mathrm{mg} / \mathrm{kg}$ dose and $-62.2 \%,-62.4 \%$, $59.1 \%,-59.3 \%$ for the $50 \mathrm{mg} / \mathrm{kg}$ dose. The administration of compound $\mathbf{9}$ at the dose of 25 $\mathrm{mg} / \mathrm{kg}$ had no significant effect on oedema formation. The higher dose of $50 \mathrm{mg} / \mathrm{kg}$ significantly decreased the oedema response at $3,4 \mathrm{~h}$ post-carrageenan by $-39.1 \%$ and $-41.1 \%$ (Fig. 2 ).

Effects of compounds 11 and 14, compound 11 given at $25 \mathrm{mg} / \mathrm{kg}$ decreased oedema formation by $43.1 \%,-40.3 \%,-34.9 \%$ at 2,3 and $4 \mathrm{~h}$ time points. 
The higher dose resulted in oedema inhibition by $49.7 \%,-42.9 \%,-35.3 \%,---31.4 \%$ at $1,2,3$ and $4 \mathrm{~h}$ time points in the study (Fig. 3). When given at doses of 25 or $50 \mathrm{mg} / \mathrm{kg}$, compound 14 inhibited oedema formation at 2,3 , $4 \mathrm{~h}$ time points. The percent inhibition was $-48.2 \%,-57.7 \%,-46.7 \%$, $35.6 \%$ for the dose of $25 \mathrm{mg} / \mathrm{kg}$ and $-47.4 \%$, $46.1 \%,-43.1 \%,-38.3 \%$ for the dose of $50 \mathrm{mg} / \mathrm{kg}$ (Fig. 3).

Effects of compounds $\mathbf{2 3}$ and 26.the administration of compound 23 resulted in significantly decreased oedema at $1,2,3,4 \mathrm{~h}$ time points post-carrageenan. The percent inhibition was $-58.3 \%,-62.7 \%,-58.8 \%$, $-52.7 \%$ for the dose of $25 \mathrm{mg} / \mathrm{kg}$ and $-58.3 \%$, $59.2 \%,-55.6 \%,-49.3 \%$ for the dose of $50 \mathrm{mg} / \mathrm{kg}$, Compound 26 given at $25 \mathrm{mg} / \mathrm{kg}$ inhibited oedema formation at 3 , 4h time points by $-31.1 \%$ and $33.4 \%$. The higher dose inhibited oedema at 2, 3, $4 \mathrm{~h}$ time points by $-35.4 \%,-36.4 \%,-38.2 \%$ (Fig. 4).

Effects of compounds $\mathbf{3 2}$ and 33, compound $\mathbf{3 2}$ given at $25 \mathrm{mg} / \mathrm{kg}$ inhibited oedema formation at 2 , 3h time points by $-35.1 \%$ and $-33.0 \%$. The higher dose inhibited the oedema response at 2, 3, $4 \mathrm{~h}$ time points by $-53 \%,-53.3 \%,-43.9 \%$. Compound 33 given at 25 or $50 \mathrm{mg} / \mathrm{kg}$ resulted in significant inhibition of the oedema response at $3,4 \mathrm{~h}$ time points by $-32.5 \%$, and $40.8 \%$ for the $25 \mathrm{mg} / \mathrm{kg}$ dose and $-32 \%$ and $33.1 \%$ for the $50 \mathrm{mg} / \mathrm{kg}$ dose (Fig.5).

The most marked and sustained inhibition of the oedema response was observed with the administration of the low and high doses of compounds $8,4,23,14$ as well as by the high dose of compound 32. There was no significant difference in the degree of oedema inhibition between the low and high dose of compounds $\mathbf{8}, \mathbf{2 3}$, $\mathbf{1 4}$ at all time points in the study. The higher dose of compound $\mathbf{4}$ was more effective in reducing oedema formation compared with the lower dose of the compound

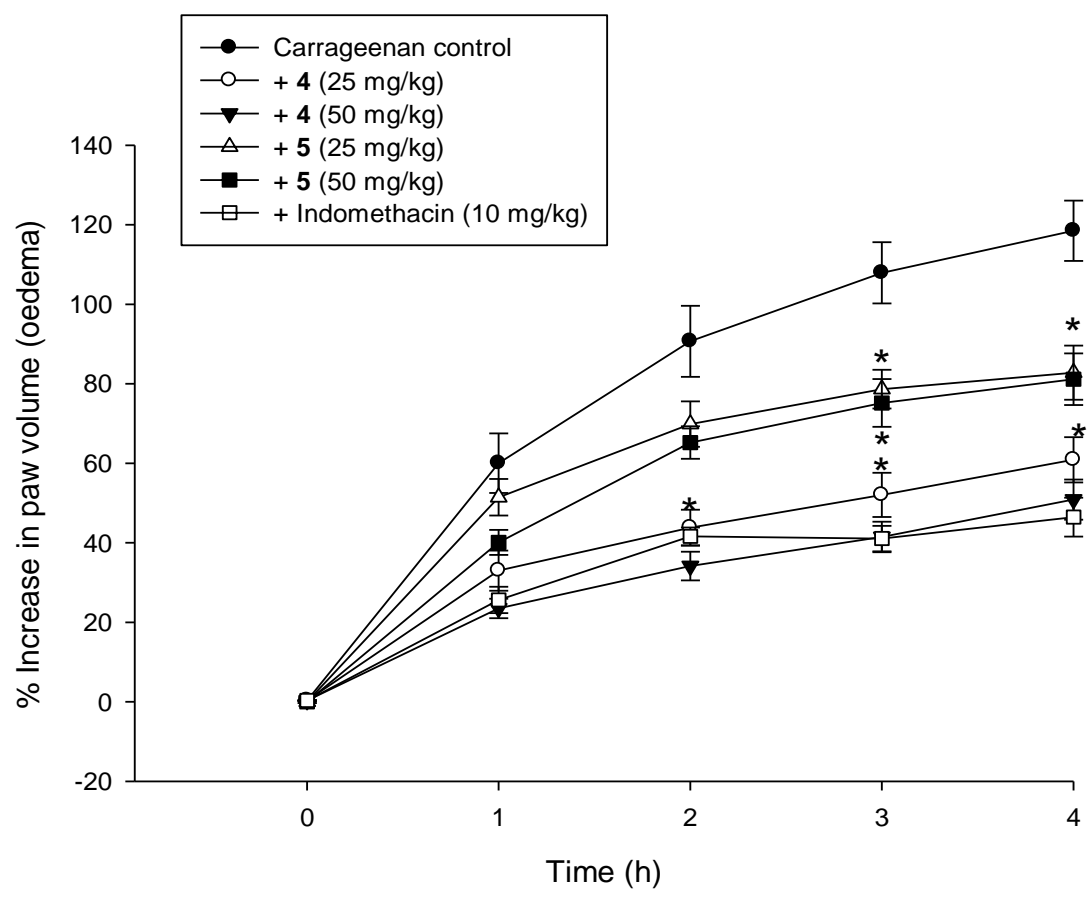

Figure 1. Effect of tested compounds $\mathbf{4}$ or $\mathbf{5}$ on the carrageenan paw oedema formation. Compounds were given $(25$ or $50 \mathrm{mg} / \mathrm{kg}$, p.o) $30 \mathrm{~min}$ prior to carrageenan injection and rats were evaluated for paw oedema at $1,2,3$ and $4 \mathrm{~h}$ post-carrageenan. Indomethacin $(10 \mathrm{mg} / \mathrm{kg}$, p.o) was used as a control drug. The results are expressed as a percentage change from control (pre-drug) values, each point represents the mean \pm S.E. of six rats per group. Asterisks indicate significant change from the carrageenan control group at the corresponding time point. 
Table 1: Effect of compounds on paw edema in carrageenan-induced inflammation

\begin{tabular}{|c|c|c|c|c|}
\hline & $1 \mathrm{~h}$ & $2 \mathrm{~h}$ & $3 \mathrm{~h}$ & $4 \mathrm{~h}$ \\
\hline Control & $60.03 \pm 7.50$ & $90.68 \pm 8.93$ & $107.9 \pm 7.70$ & $118.5 \pm 7.60$ \\
\hline (4) $25 \mathrm{mg} / \mathrm{kg}$ & $32.99 \pm 5.05$ & $43.78 \pm 4.53 *$ & $52.03 \pm 5.56^{*}$ & $60.87 \pm 5.71^{*}$ \\
\hline (4) $50 \mathrm{mg} / \mathrm{kg}$ & $23.48 \pm 2.46^{*}$ & $34.13 \pm 3.61 *$ & $41.46 \pm 3.84 *$ & $50.87 \pm 5.06^{*}$ \\
\hline (5) $25 \mathrm{mg} / \mathrm{kg}$ & $51.44 \pm 4.60$ & $69.86 \pm 5.71$ & $78.65 \pm 4.89$ & $82.79 \pm 6.80 *$ \\
\hline (5) $50 \mathrm{mg} / \mathrm{kg}$ & $40.09 \pm 3.14$ & $65.21 \pm 4.10$ & $75.17 \pm 6.02 *$ & $81.13 \pm 6.5^{*}$ \\
\hline (8) $25 \mathrm{mg} / \mathrm{kg}$ & $22.49 \pm 1.52 *$ & $29.14 \pm 0.64 *$ & $37.74 \pm 1.25^{*}$ & $43.70 \pm 2.14 *$ \\
\hline (8) $50 \mathrm{mg} / \mathrm{kg}$ & $22.70 \pm 1.03 *$ & $34.07 \pm 2.70 *$ & $44.14 \pm 3.41 *$ & $48.21 \pm 3.49 *$ \\
\hline (9) $25 \mathrm{mg} / \mathrm{kg}$ & $47.25 \pm 4.20$ & $72.71 \pm 6.61$ & $81.42 \pm 6.50$ & $89.42 \pm 5.41$ \\
\hline (9) $50 \mathrm{mg} / \mathrm{kg}$ & $47.73 \pm 3.57$ & $61.02 \pm 1.88$ & $65.76 \pm 2.18^{*}$ & $69.76 \pm 4.25^{*}$ \\
\hline (11) $25 \mathrm{mg} / \mathrm{kg}$ & $38.92 \pm 2.85$ & $51.63 \pm 3.37 *$ & $64.44 \pm 4.90 *$ & $77.15 \pm 5.13 *$ \\
\hline (11) $50 \mathrm{mg} / \mathrm{kg}$ & $30.16 \pm 2.51 *$ & $51.70 \pm 4.22$ & $69.79 \pm 4.00 *$ & $81.24 \pm 5.06^{*}$ \\
\hline (14) $25 \mathrm{mg} / \mathrm{kg}$ & $31.11 \pm 2.14$ & $43.83 \pm 3.00 *$ & $57.46 \pm 2.95 *$ & $76.25 \pm 4.18 *$ \\
\hline (14) $50 \mathrm{mg} / \mathrm{kg}$ & $31.60 \pm 1.75$ & $48.86 \pm 4.03 *$ & $61.34 \pm 2.38 *$ & $73.15 \pm 3.11 *$ \\
\hline (23) $25 \mathrm{mg} / \mathrm{kg}$ & $25.02 \pm 2.08 *$ & $33.77 \pm 3.15^{*}$ & $44.42 \pm 2.13^{*}$ & $56.02 \pm 3.2 *$ \\
\hline (23) $50 \mathrm{mg} / \mathrm{kg}$ & $24.95 \pm 2.00 *$ & $37.02 \pm 2.61 *$ & $47.86 \pm 4.60 *$ & $60.06 \pm 5.18^{*}$ \\
\hline (26) $25 \mathrm{mg} / \mathrm{kg}$ & $46.29 \pm 2.26$ & $65.53 \pm 3.47$ & $74.32 \pm 3.70^{*}$ & $78.95 \pm 4.90 *$ \\
\hline (26) $50 \mathrm{mg} / \mathrm{kg}$ & $41.74 \pm 2.72$ & $58.57 \pm 3.14 *$ & $68.58 \pm 4.18^{*}$ & $73.28 \pm 3.11 *$ \\
\hline (32) $25 \mathrm{mg} / \mathrm{kg}$ & $35.44 \pm 2.64$ & $58.87 \pm 4.10 *$ & $72.26 \pm 6.32 *$ & $86.95 \pm 4.50$ \\
\hline (32) $50 \mathrm{mg} / \mathrm{kg}$ & $36.06 \pm 3.15$ & $42.62 \pm 2.80 *$ & $61.14 \pm 5.23^{*}$ & $66.50 \pm 5.20 *$ \\
\hline (33) $25 \mathrm{mg} / \mathrm{kg}$ & $42.27 \pm 3.48$ & $65.94 \pm 3.68$ & $72.81 \pm 5.30 *$ & $70.12 \pm 4.00 *$ \\
\hline (33) $50 \mathrm{mg} / \mathrm{kg}$ & $44.16 \pm 2.93$ & $72.52 \pm 3.50$ & $73.35 \pm 6.98 *$ & $79.22 \pm 5.14 *$ \\
\hline $\begin{array}{l}\text { Indomethacin } 10 \\
\mathrm{mg} / \mathrm{kg}\end{array}$ & $25.62 \pm 3.30 *$ & $41.62 \pm 2.24 *$ & $41.05 \pm .3 .21 *$ & $46.45 \pm 4.89 *$ \\
\hline
\end{tabular}

Data are expressed as mean \pm SE. $n=6$ rats per group. The values indicate the percentage increase of paw volume (oedema) from control (basal pre-drug) values. Drugs were, orally administered 30 min before the injection of carrageenan. Statistically significant from vehicle control at corresponding time point: $* \mathrm{P}<0.05$. 


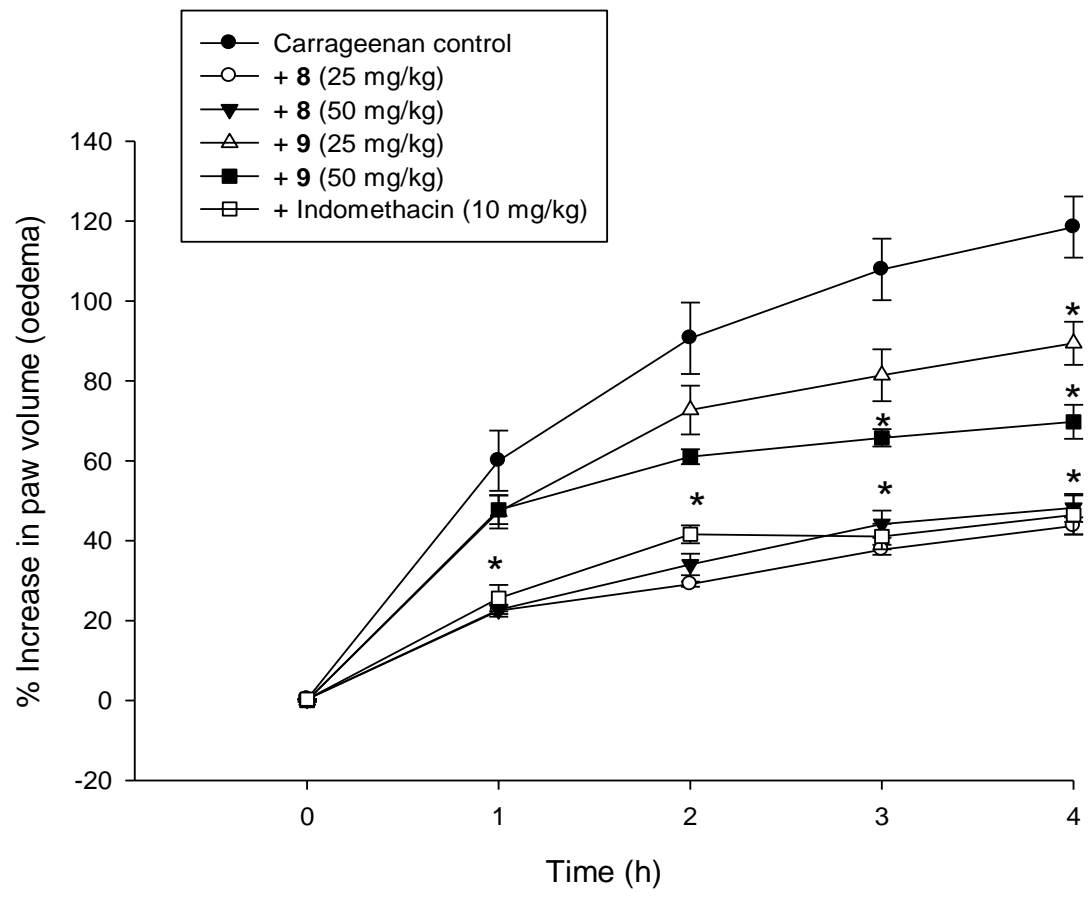

Figure 2. Effect of tested compounds $\mathbf{8}$ or $\mathbf{9}$ on the carrageenan paw oedema formation. Compounds were given ( 25 or $50 \mathrm{mg} / \mathrm{kg}$, p.o.) $30 \mathrm{~min}$ prior to carrageenan injection and rats were evaluated for paw oedema at 1, 2, 3 and $4 \mathrm{~h}$ post-carrageenan. Indomethacin $(10 \mathrm{mg} / \mathrm{kg}$, p.o.) was used as a control drug. The results are expressed as a percentage change from control (pre-drug) values, each point represents the mean \pm S.E. of six rats per group. Asterisks indicate significant change from the carrageenan control group at the corresponding time point.

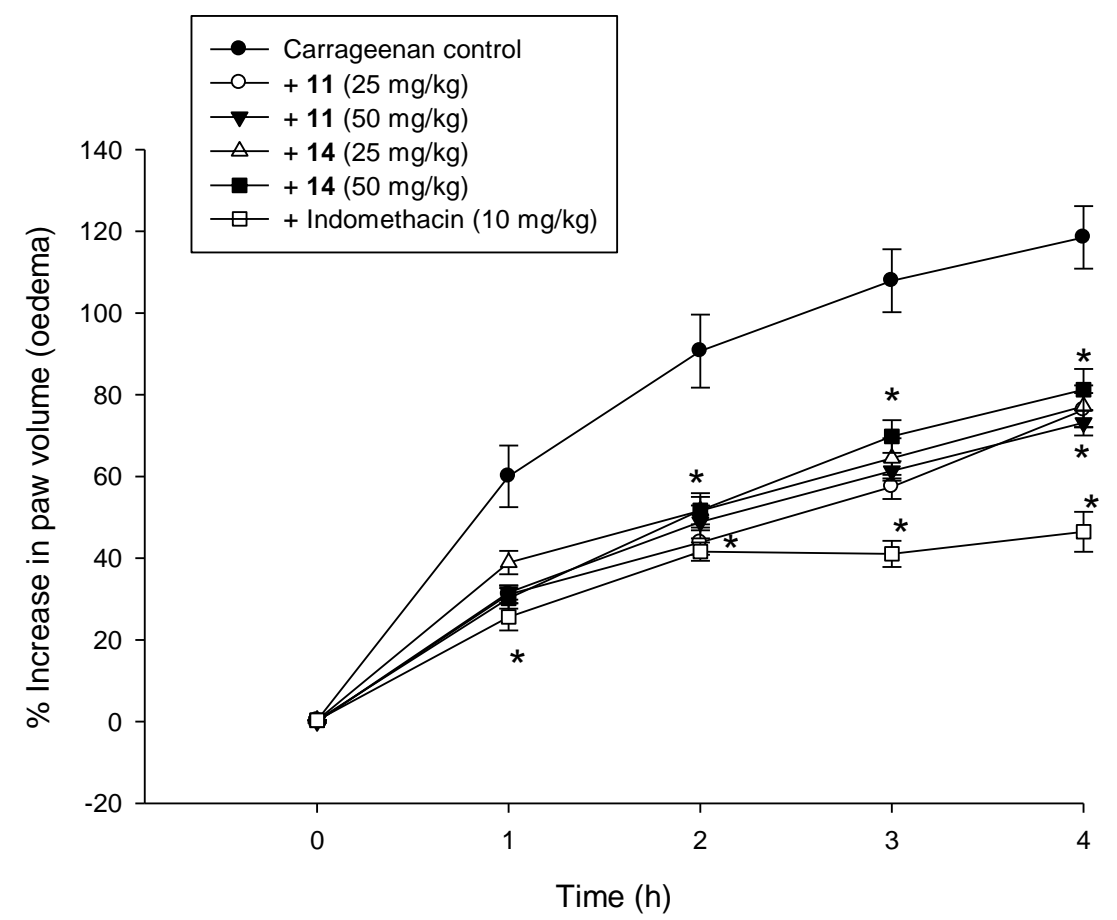

Figure 3. Effect of tested compounds $\mathbf{1 1}$ or $\mathbf{1 4}$ on the carrageenan paw oedema formation. Compounds were given ( 25 or $50 \mathrm{mg} / \mathrm{kg}$, p.o.) $30 \mathrm{~min}$ prior to carrageenan injection and rats were evaluated for paw oedema at 1, 2, 3 and $4 \mathrm{~h}$ post-carrageenan. Indomethacin $(10 \mathrm{mg} / \mathrm{kg}$, p.o.) was used as a control drug. The results are expressed as a percentage change from control (pre-drug) values, each point represents the mean \pm S.E. of six rats per group. Asterisks indicate significant change from the carrageenan control group at the corresponding time point. 




Figure 4. Effect of tested compounds $\mathbf{2 3}$ or $\mathbf{2 6}$ on the carrageenan paw oedema formation. Compounds were given ( 25 or $50 \mathrm{mg} / \mathrm{kg}$, p.o.) $30 \mathrm{~min}$ prior to carrageenan injection and rats were evaluated for paw oedema at 1, 2, 3 and $4 \mathrm{~h}$ post-carrageenan. Indomethacin $(10 \mathrm{mg} / \mathrm{kg}$, p.o.) was used as a control drug. The results are expressed as a percentage change from control (pre-drug) values, each point represents the mean \pm S.E. of six rats per group. Asterisks indicate significant change from the carrageenan control group at the corresponding time point.

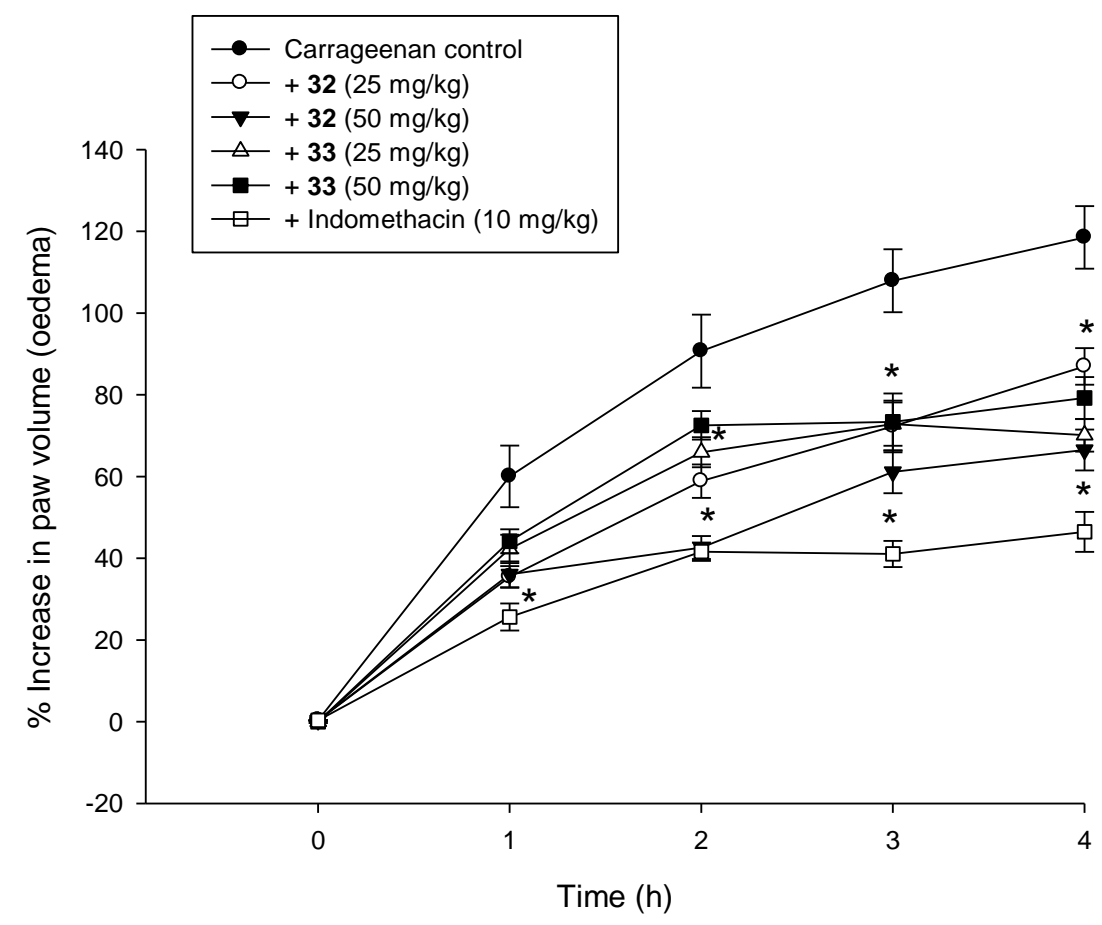

Figure 5. Effect of tested compounds $\mathbf{3 2}$ or $\mathbf{3 3}$ on the carrageenan paw oedema formation. Compounds were given ( 25 or $50 \mathrm{mg} / \mathrm{kg}$, p.o.) $30 \mathrm{~min}$ prior to carrageenan injection and rats were evaluated for paw oedema at 1, 2, 3 and $4 \mathrm{~h}$ post-carrageenan. Indomethacin $(10 \mathrm{mg} / \mathrm{kg}$, p.o.) was used as a control drug. The results are expressed as a percentage change from control (pre-drug) values, each point represents the mean \pm S.E. of six rats per group. Asterisks indicate significant change from the carrageenan control group at the corresponding time point. 


\subsubsection{Antiulcer effects}

In order to test the effect of the tested compounds on the gastric mucosa, we investigated their effect on the development of gastric mucosal lesions induced in the rat by the intragastric administration of $96 \%$ ethanol in comparison with Ranitidine $(50 \mathrm{mg} / \mathrm{kg}$ ). Rats were fasted for $18 \mathrm{~h}$ but allowed water ad libitum. They were orally administered either saline (control) or the test compounds $30 \mathrm{~min}$ prior to ethanol $(96 \%, 1 \mathrm{~mL}$, p.o.). Each compound was injected orally in two doses $(25$ or $50 \mathrm{mg} / \mathrm{kg}$ ). It was found that gastric mucosal lesions caused by $96 \%$ ethanol were inhibited by all tested compounds in the study. Compounds 4, 9, 11, 26, 32 were the most effective in inhibiting lesion formation. No lesions were observed after the administration of compounds $\mathbf{9}$, 26 at $50 \mathrm{mg} / \mathrm{kg}$ (Table 2).

\section{Structure activity relationship}

The analysis of the structure activity relationships indicates first that the cytotoxicity of the tested compounds seems to be linked to the incorporation of the heterocyclic ring to the steroid moiety. The antiinflammatory activity of the thiophene steroids 4,14 and $\mathbf{2 3}$ are more than that of the other thiophene steroid derivatives, this may attribute to the ethyl carboxylate group of compound $\mathbf{4}$, benzoyl group of compound $\mathbf{1 4}$ and phenylamino group of compound 23 in addition to the amino groups and other pharmacophoric feature of the thiophene and steroid moieties. The addition of diamino-oxopyridine moiety to form compound $\mathbf{8}$ is more potent than the addition of other heterocycles. The consequence of antiulcer effects showed distinguished results of the newly synthesized hetero-steroids as they inhibit lesion formation. The presence of ethyl carboxylatedihydrodiamino pyridine moiety in compound $\mathbf{9}$ and the presence of aminophenylthioxothiazole moiety in compound 26 in addition to the pharmacophoric feature of the steroid moiety observed no lesions formation at $50 \mathrm{mg} / \mathrm{kg}$. The results assured that incorporation of heterocyclic rings into steroid moiety enhances the activity. Beside that compounds containing sulfur and nitrogen in the heterocyclic ring have an obvious anti-inflammatory effect.

\section{Conclusion}

This research outlined the synthesis of novel hybrid heterocyclic steroids via straight forward and effective methods. The newly synthesized altered steroids showed different intensities of antiinflammatory and anti-ulcerogenic activity. The most marked and sustained inhibition of the oedema response was observed with the administration of the low and high doses of compounds $8,4,23,14$ as well as by the high dose of compound 32. Gastric mucosal lesions induced by $96 \%$ ethanol in the rat were substantially inhibited by either of the compounds under investigation. These findings offer a distinctive chance to create fresh antiinflammatory drugs that avoid ulcerative liabilities associated with drugs presently on the market. Finally, in the hope of discovering fresh powerful prescriptions, promoting outcomes of antiinflammatory and anti-ulcerogenic activity shown by these compounds may be of concern for further derivation and further drug ability and physical research

\section{Declaration of interest}

The authors declare that they have no conflict of interest

\section{Acknowledgements}

The authors acknowledge the financial support of the National Research Centre, Cairo, Egypt: grant no.10010105 for Prof Gamal A. Elmegeed and grant no. 11010335 for Dr. Mohamed A. Tantawy. 
Table 2: Ulcer number and severity of ethanol -induced gastric lesions in rats treated with different compounds.

\begin{tabular}{|c|c|c|}
\hline & Ulcer number & Ulcer severity \\
\hline Normal & $0.00 \pm 0.00$ & $0.00 \pm 0.00$ \\
\hline Ethanol & $18.40 \pm 1.69$ & $27.40 \pm 1.57$ \\
\hline Ranitidine 50 mg/kg & $\begin{array}{l}1.40 \pm 0.12 * \\
(-92.4 \%)\end{array}$ & $\begin{array}{l}2.02 \pm 0.15^{*} \\
(-92.6 \%)\end{array}$ \\
\hline (4) $25 \mathrm{mg} / \mathrm{kg}$ & $\begin{array}{l}3.80 \pm 0.19 *+ \\
(-79.3 \%)\end{array}$ & $\begin{array}{l}8.20 \pm 0.12 *+ \\
(-70.1 \%)\end{array}$ \\
\hline (4) $50 \mathrm{mg} / \mathrm{kg}$ & $\begin{array}{l}1.20 \pm 0.58 * \\
(-93.4 \%)\end{array}$ & $\begin{array}{l}2.40 \pm 0.09 * \\
(--91.2 \%)\end{array}$ \\
\hline (5) $25 \mathrm{mg} / \mathrm{kg}$ & $\begin{array}{l}4.20 \pm 0.61 *_{+} \\
(-77.2 \%)\end{array}$ & $\begin{array}{l}18.20 \pm 0.92 *+ \\
(-33.6 \%)\end{array}$ \\
\hline (5) $50 \mathrm{mg} / \mathrm{kg}$ & $\begin{array}{l}4.40 \pm 0.50 *+ \\
(-76.1 \%)\end{array}$ & $\begin{array}{l}13.60 \pm 0.97 * \\
(-50.4 \%)\end{array}$ \\
\hline (8) $25 \mathrm{mg} / \mathrm{kg}$ & $\begin{array}{l}4.20 \pm 0.74 *_{+} \\
(-77.2 \%)\end{array}$ & $\begin{array}{l}9.20 \pm 0.12 * \\
(-66.4 \%)\end{array}$ \\
\hline (8) $50 \mathrm{mg} / \mathrm{kg}$ & $\begin{array}{l}2.20 \pm 0.02 *+ \\
(-88.0 \%)\end{array}$ & $\begin{array}{l}4.00 \pm 0.31{ }^{*}+ \\
(-85.4 \%)\end{array}$ \\
\hline (9) $25 \mathrm{mg} / \mathrm{kg}$ & $\begin{array}{l}1.00 \pm 0.24 * \\
(-94.6 \%)\end{array}$ & $\begin{array}{l}2.67 \pm 0.17 * \\
(-90.2 \%)\end{array}$ \\
\hline (9) $50 \mathrm{mg} / \mathrm{kg}$ & $\begin{array}{l}0.00 \pm 0.00 *+ \\
(-100 \%)\end{array}$ & $\begin{array}{l}0.00 \pm 0.00 *+ \\
(-100.0 \%)\end{array}$ \\
\hline (11) $25 \mathrm{mg} / \mathrm{kg}$ & $\begin{array}{l}4.40 \pm 0.50 *+ \\
(-76.1 \%)\end{array}$ & $\begin{array}{l}12.00 \pm 1.65^{*}+ \\
(-56.2 \%)\end{array}$ \\
\hline (11) $50 \mathrm{mg} / \mathrm{kg}$ & $\begin{array}{l}0.75 \pm 0.47 * \\
(-96 \%)\end{array}$ & $\begin{array}{l}1.25 \pm 0.09 * \\
(-95.4 \%)\end{array}$ \\
\hline (14) $25 \mathrm{mg} / \mathrm{kg}$ & $\begin{array}{l}5.60 \pm 0.22 *_{+} \\
(-69.6 \%)\end{array}$ & $\begin{array}{l}12.60 \pm 0.21 *_{+} \\
(-54.0 \%)\end{array}$ \\
\hline (14) $50 \mathrm{mg} / \mathrm{kg}$ & $\begin{array}{l}2.80 \pm 0.25^{*}+ \\
(-84.8 \%)\end{array}$ & $\begin{array}{l}4.20 \pm 0.21{ }^{*}+ \\
(-84.7 \%)\end{array}$ \\
\hline (23) $25 \mathrm{mg} / \mathrm{kg}$ & $\begin{array}{l}4.00 \pm 0.04 * \\
(-78.3 \%)\end{array}$ & $\begin{array}{l}14.00 \pm 0.23 * \\
(-49.0 \%)\end{array}$ \\
\hline (23) $50 \mathrm{mg} / \mathrm{kg}$ & $\begin{array}{l}4.50 \pm 0.09 *+ \\
(-75.5 \%)\end{array}$ & $\begin{array}{l}7.25 \pm 0.25 *+ \\
(-73.5 \%)\end{array}$ \\
\hline (26) $25 \mathrm{mg} / \mathrm{kg}$ & $\begin{array}{l}1.00 \pm 0.08 * \\
(-94.6 \%)\end{array}$ & $\begin{array}{l}1.40 \pm 0.06^{*} \\
(-95.0 \%) \\
\end{array}$ \\
\hline (26) $50 \mathrm{mg} / \mathrm{kg}$ & $\begin{array}{l}0.00 \pm 0.00 *+ \\
(-100.0 \%)\end{array}$ & $\begin{array}{l}0.00 \pm 0.00 *_{+} \\
(-100.0 \%)\end{array}$ \\
\hline (32) $25 \mathrm{mg} / \mathrm{kg}$ & $\begin{array}{l}3.75 \pm 0.35^{*} \\
(-79.6 \%)\end{array}$ & $\begin{array}{l}7.50 \pm 0.61 * \\
(-72.6 \%)\end{array}$ \\
\hline (32) $50 \mathrm{mg} / \mathrm{kg}$ & $\begin{array}{l}1.40 \pm 0.09 * \\
(-92, .4 \%)\end{array}$ & $\begin{array}{l}2.00 \pm 0.14 * \\
(-92.7 \%)\end{array}$ \\
\hline (33) $25 \mathrm{mg} / \mathrm{kg}$ & $\begin{array}{l}5.80 \pm 0.23 *_{+} \\
(-68.5 \%)\end{array}$ & $\begin{array}{l}16.60 \pm 0.80 *+ \\
(-39.4 \%)\end{array}$ \\
\hline (33) $50 \mathrm{mg} / \mathrm{kg}$ & $\begin{array}{l}3.00 \pm 0.36 *+ \\
(-83.7 \%)\end{array}$ & $\begin{array}{l}8.60 \pm 1.25 *+ \\
(-68.6 \%)\end{array}$ \\
\hline
\end{tabular}

Results are mean \pm S.E.M. $(\mathrm{n}=6)$. Statistical comparison was performed by using ANOVA followed by Mann Whitney test. * $\mathrm{P}<0.05$ were consider statistically significant when compared to ethanol control group. $+\mathrm{P}<0.05$ were consider statistically significant when compared to ranitidine treated group. the perecent inhibition of lesion number or severity compared to the control group is shown in parenthesis. 


\section{References}

Albuquerque, H.M.T., Santos, C.M.M., Silva, A.M.S. (2019). Cholesterol-based compounds: Recent advances in synthesis and applications. J. Molecules., 24(1): 116

Ansari, A., Ali, A., Asif, M., Shamsuzzaman. (2019) Steroidal thiazolidinone derivatives: Design, synthesis and their molecular interaction with human serum albumin. J. Steroids., 148, 99113.

Cai, X., Zhao, S., Cai., Zheng, J., Zhu, Z., Wei, D., Zheng, Z., Zhu, H., Chen, Y. (2019). Synthesis and evaluation of novel D-ring substituted steroidal pyrazolines as potential anti-inflammatory agents. Steroids., 146: 708.

Doss, S.H., Louca, N.A., Elmegeed, G.A., Mohareb, R.M. (1999). Sultamo-Steroid Analogues, Part VII: Synthesis of epi-Androsterone derivatives with potential activity. Arch. Pharm. Res., 22(5), 496-501.

Elgazwy, A.H, Nassar, E., Zaki, M. (2012) Synthesis, biological evaluation of some 2,3dihydropyrazoles and thiazoles as antiinflammatory and antibacterial agents. J. Org. Chem. Curr. Res., 1(112): 2161.

Elmegeed, G.A., Baiuomy, A.R., Abdelhalim, M.M., Hana H.Y.(2010). Synthesis and antidepressant evaluation of five novel heterocyclic tryptophan-hybrid derivatives. J. Arch. Pharm. Chem. Life Sci., 343(5): 26167.

Elmegeed, G.A., Baiuomy, A.R., Abdel-Salam, O.M. (2007). Evaluation of the antiinflammatory and anti-nociceptive activities of novel synthesized melatonin analogues. Eur. J. Med. Chem., 42(10): 1285-92.

Elmegeed, G.A., Wardakhan, W.W., Baiuomy, A. (2005). Synthesis of thiazolyl and thieno cholestane derivatives: a novel class of potent antiinflammatory steroids. J Pharmazie., 60(5): 328-33.

Frank, É., Schneider, G. (2013). Synthesis of sex hormone-derived modified steroids possessing antiproliferative activity, J. Steroid Biochem. \& Molecul. Biol., 137: 301-315.

Fuente, A., Reyes, M., Alvarez, Y.M., Ruiz, J.A., Vélez, H., Viñas-Bravo, O., Montiel-Smith, S., Meza-Reyes, S., Sandoval-Ramírez J.(2005). $\quad{ }^{1} \mathrm{H}$ and ${ }^{13} \mathrm{C}$ NMR spectral assignment of androstane derivatives. J. Magn. Reson. Chem., 43: 676-78.

Kozuch, P.L., Hanauer, S.B. (2008). Treatment of inflammatory bowel disease: a review of medical therapy. World J Gastroenterol., 14(3): 354-77.

Ledneczk,i I., Némethy, Z., Tapolcsányi, P., Éles, J., Greiner, I., Gábor, E., Varga, B., Balázs, O., Román, V., Lévay, G., Mahó, S. (2019). Discovery of novel steroidal histamine $\mathrm{H} 3$ receptor antagonists/inverse agonists. Part2. Versatile steroidal carboxamide derivatives. J. Bioorg. \& Med. Chem, Lett., 29(20), 126643.

Lipworth, B.J.(1999). Systemic adverse effects of inhaled corticosteroid therapy: a systematic review and meta-analysis. J. Arch. Internal Medicine., 159(9): 941-55.

Mohareb, R.M., Elmegeed, G.A., Baiuomy, A.R., Eskander, E. F. William, M.G. (2011). Evaluation of anti-inflammatory, antinociceptive, and anti-ulcerogenic activities of novel Synthesized thiazolyl and pyrrolyl steroids. J. Arch. Pharm. Chem. Life Sci., 344(9): 595-604.

Mózsik, G.Y., Móron, F., Jávor, T. (1982). Cellular mechanisms of the development of gastric mucosal damage and of gastrocytoprotection induced by prostacyclin in rats. A pharmacological study. J. Prostaglandins Leukot Med., 9(1): 71-84.

Naumann, J.M., Zöllner, A., Drăgan, C.A., Messinger, J., Adam, J., \& Bureik, M. (2011) Biotechnological production of 20-alphadihydrodydrogesterone at pilot scale. J. Appl. Biochem. Biotech., 165(1): 190-203.

NIH publication No. 85-23, revised 1985. Pharmazie., 60, 328. 
Pillai, A.D., Rathod, P.D., P X F , Patel, M., Nivsarkar, M., Vasu K.K., Padh, H., Sudarsanam, V. (2003). Novel drug designing approach for dual inhibitors as antiinflammatory agents: implication of pyridine template. J. Biochem. and Biophys. Res. Comm., 301(1): 183-86.

Soni, J.P., Sen, D.J., Modh, K.M. (2011). Structure activity relationship studies of synthesized pyrazolone derivatives of imidazole, benzimidazole and benztriazole moiety for anti-inflammatory activity. J Appl. Pharmaceut. Sci., 1(4): 115-120.

Tantawy, M.A., Nafie, M. S., Elmegeed, G.A. (2019). Screening of different drug design tools to predict the mode of action of steroidal derivatives as anti-cancer agents. J. Steroids., $152,108485$.

Venkatachalam, S.R., Salaskar, A., Chattopadhyay, A., Barik, A., Mishra, B., Gangabhagirathi, R., Priyadarsini, K.I. (2006). Synthesis, pulse radiolysis, and in vitro radioprotection studies of melatoninolipoamide, a novel conjugate of melatonin and $\alpha$-lipoic acid. Bioorg. \& Med. Chem., 14(18): 6414 -19.

Winter, C.A., Risley, E.A., Nuss, G.W. (1962). Carrageenin-induced edema in hind paw of the rat as an assay for antiinflammatory drugs. Proc Soc Exp. Biol Med., 111(3): 5445-547. 